\title{
RESEARCH
}

\section{Amplicon Sequencing of Fusarium Translation Elongation Factor $1 \alpha$ Reveals that Soil Communities of Fusarium Species Are Resilient to Disturbances Caused by Crop and Tillage Practices}

\author{
Peter M. Henry, ${ }^{1,+(D)}$ Samuel I. Koehler, ${ }^{1}$ Sukhwinder Kaur, ${ }^{2}$ Lynn Epstein, ${ }^{2} \mathbb{D}$ Jeffrey P. Mitchell, ${ }^{3}$ Thomas R. Gordon, ${ }^{2,4}(\mathbb{D}$ and \\ Johan H. J. Leveau ${ }^{2}$ (D) \\ ${ }^{1}$ United States Department of Agriculture-Agricultural Research Service, 1636 E. Alisal St., Salinas, CA 93905 \\ 2 Department of Plant Pathology, University of California, One Shields Avenue, Davis, CA 95616 \\ ${ }^{3}$ Department of Plant Sciences, University of California, One Shields Avenue, Davis, CA 95616 \\ ${ }^{4}$ Deceased 27 June 2021. \\ Accepted for publication 13 February 2022.
}

\section{ABSTRACT}

The fungal genus Fusarium contains plant pathogens, mutualists, and commensalists. Because nonpathogenic Fusarium strains can contribute to disease suppression in soil by competition or induced plant resistance, identifying factors that influence the abundance of nonpathogenic strains could lead to strategies that enhance plant-beneficial interactions and deter pathogen invasion. We developed a Fusarium-specific high-throughput translation elongation factor $1 \alpha$ (EF-1 $\alpha)$ amplicon-sequencing method with subspecies resolution. Newly designed primers enabled reads to be joined for improved quality metrics after Illumina MiSeq sequencing. By including the 'oligotyping' program in our bioinformatic workflow, we reconstructed Fusarium EF-1 $\alpha$ haplotypes with $100 \%$ accuracy. We conducted simulated crop rotation experiments in soil infested with the strawberry pathogen Fusarium oxysporum f. sp. fragariae (Fof) and the following treatments: strawberry, blackberry, raspberry, lettuce, broccoli, and fallow. We profiled root Fusarium communities with EF-1 $\alpha$ amplicons and observed that nonpathogenic strains of $F$. oxysporum did not colonize crops uniformly; some strains exhibited asymptomatic host preferences. In susceptible strawberry roots, $>90 \%$ of sequences corresponded to Fof. Bulk soil communities of Fusarium were remarkably stable and had minimal treatment-dependent effects after 1 year. Similarly, in a long-term agricultural research experiment, 18 years of conservation tillage and cover cropping did not lead to bulk soil Fusarium communities that were significantly different than controls. Although the roots of rotation crops can augment the abundance of nonpathogenic F. oxysporum strains, their abundance in bulk soil is unlikely to be altered after a single year. Climate and soil physicochemical properties may have a greater effect on Fusarium community structure.

Keywords: amplicon sequence variants, conservation tillage, cover cropping, fungal communities, Fusarium wilt
Reproduction on susceptible plant tissues contributes to the persistence of many soilborne pathogens (Henry et al. 2019a; Mol et al. 1995; Singh et al. 1990; Xiao et al. 1998). This lifecycle

Corresponding author: P. M. Henry; peter.henry@usda.gov

Funding: Thanks to our funding sources: the National Science Foundation Graduate Research Fellowship Program, the California Strawberry Commission, and the National Institute of Food and Agriculture (NIFA) Specialty Crops Research Initiative (grant number 2017-51181-26833)

$\boldsymbol{e}$-Xtra: Supplementary materials and supplementary datasets are available online. The author(s) declare no conflict of interest.

Copyright (c) 2022 The Author(s). This is an open access article distributed under the CC BY-NC-ND 4.0 International license. process can be disrupted by planting rotation crops that are unfavorable for pathogen population growth (Henry et al. 2019a; Singh et al. 1990; Xiao et al. 1998). Rotation crop cultivation and decomposition can also influence the taxonomic composition of soil microbiota (Peralta et al. 2018), whose interactions with pathogenic organisms may alter disease outcomes (Siegel-Hertz et al. 2018; Weller et al. 2002). Superior disease control can be achieved with crop rotations that both avoid pathogen proliferation and augment natural populations of disease suppressive organisms (Inderbitzin et al. 2018).

Control of Fusarium wilt diseases through crop rotation is limited by the pathogen's ability to asymptomatically colonize a wide diversity of plant species and compete saprotrophically for organic matter in soil (Gordon and Okamoto 1990; Gordon et al. 1989; Henry et al. 2019a). Thus, these pathogens can persist in soil, de- 
spite decreasing in abundance during periods without symptomatic hosts (Henry et al. 2019a; Scott et al. 2012). Fusarium wilt diseases are caused by host-specific pathogenic strains of Fusarium oxysporum (called 'formae speciales'), and at least 106 formae speciales have been reported (Edel-Hermann and Lecompte 2019). In addition to plant pathogens, the $F$. oxysporum species complex harbors a great diversity of apparently nonpathogenic strains (Gordon 2017), some of which possess biological control capabilities for Fusarium wilt diseases (Bolwerk et al. 2005; Constantin et al. 2019; de Lamo et al. 2020). Although unfeasible to prove experimentally that an isolate cannot cause disease on any plant, it is generally accepted that there are truly nonpathogenic strains (Demers et al. 2015; Gordon and Martyn 1997). Multiple lines of evidence support this assumption: diverse $F$. oxysporum strains are frequently isolated from healthy plants (Gordon et al. 1989), and lack genomic signatures of pathogenicity (Constantin et al. 2021). Nonpathogenic F. oxysporum strains are ubiquitous in soil and are almost always present in the same locations as pathogenic strains (Bao et al. 2004; Demers et al. 2015; Gordon and Martyn 1997). There is evidence that nonpathogenic strains of F. oxysporum and other Fusarium spp. contribute to specific suppression of Fusarium wilt diseases (Alabouvette 1986; Schneider 1984; Siegel-Hertz et al. 2018).

Although past research has identified niches that are important for the persistence of $F$. oxysporum, we lack an understanding of how these processes manifest at a community level to affect the abundance of individual strains in the natural environment. For example, once a pathogen establishes in soil where its susceptible host is grown, does it outcompete resident nonpathogenic strains and become the dominant strain in that location? Or, are there community dynamics that limit pathogen abundance after introduction? In addition, what are the relative contributions of endophytic growth on living plant roots and saprotrophic growth on soil organic matter for the survival of nonpathogenic or pathogenic F. oxysporum? Past research indicates there can be host specialization for asymptomatic plant colonization (Dhingra and Coelho Netto 2001; Haware and Nene 1982; Henry et al. 2019a) but is this broadly relevant among presumed nonpathogenic strains or an uncommon feature of some pathogens? Dissecting these questions could lead to new management strategies that harness the biocontrol potential of nonpathogenic strains and discern the fundamental mechanisms that limit pathogen abundance in soil.

To start answering these questions, a high-throughput method is needed to determine the abundance of pathogenic strains of F. oxysporum in context with the resident nonpathogenic strains of Fusarium and non-Fusarium fungi. Fungal communities are typically characterized by high-throughput sequencing of internal transcribed spacer (ITS) amplicons and taxonomic classification of the resulting reads (Bokulich and Mills 2013; Taylor et al. 2016). However, ITS amplicon sequencing only discriminates to the genus or species level (Bokulich and Mills 2013) and, therefore, it is limited in its ability to provide meaningful data for genus Fusarium, where ecological differences at the subspecies level are common (Gordon and Martyn 1997; O’Donnell et al. 2012).

An intron-rich region of the translation elongation factor $1 \alpha$ (EF-1 $\alpha$ ) gene provides better phylogenetic resolution within Fusarium than ITS (O'Donnell et al. 1998). This locus is widely used for phylogenetic comparisons and many reference sequences are available from public databases (O'Donnell et al. 2009). Because of these characteristics, Fusarium-specific high-throughput EF-1 $\alpha$ amplicon sequencing methods were developed (Boutigny et al. 2019; Cobo-Díaz et al. 2019; Karlsson et al. 2016). However, the amplicons generated by these protocols are either (i) 500 to 700 bp long and cannot be reliably joined into a single sequence from paired-end reads on the commonly used Illumina MiSeq platform (Boutigny et al. 2019; Karlsson et al. 2016) or (ii) include significant proportions of exon sequence with fewer phylogenetically informative sites (Cobo-Díaz et al. 2019). Because joining paired-end reads into a single sequence can greatly improve quality scores, most protocols for ITS and 16S-based community profiling use amplicons that can be joined after paired-end sequencing and target variable, noncoding regions (Bokulich and Mills 2013; Taylor et al. 2016). These modifications could improve existing protocols and facilitate implementation with currently available sequencing technology.

The objectives of this study were to (i) design a new primer pair and protocol for Fusarium EF-1 $\alpha$ amplicon-sequencing that is compatible with the Illumina MiSeq platform and that allows paired-end joining and (ii) apply this tool to investigate the impact of crop rotation, cover cropping, and tillage practices on the relative abundance of Fusarium amplicon sequence variants (ASVs). To achieve these objectives, we developed primers to specifically amplify an approximately 400- to 450-bp EF-1 $\alpha$ amplicon from Fusarium spp. that contains $>80 \%$ intron regions and can be joined into a single sequence from Illumina MiSeq paired-end reads. We analyzed reads generated from this protocol with a novel bioinformatics workflow that yielded subspecies-level relative abundances of Fusarium ASVs from environmental samples. We applied our method to investigate the colonization of rotation crops by Fusarium ASVs in field soil that was artificially infested with $F$. oxysporum f. sp. fragariae (Fof), the cause of Fusarium wilt of strawberry, and determined how changes in the soil inoculum density of this pathogenic strain related to other Fusarium ASVs. In addition, we analyzed the impact of an 18-year conservation tillage and cover cropping experiment on bulk soil and root endosphere assemblies of Fusarium ASVs. The outcome of this study is a detailed view of Fusarium community dynamics in agricultural systems.

\section{MATERIALS AND METHODS}

Fusarium EF-1 $\alpha$ reference database construction. Two databases were constructed: the first was a database of 2,726 manually curated sequences that we used for taxonomic classification, and the second was a broad survey of 21,159 sequences from NCBI GenBank that were used for primer design and validation.

To construct the first database, we compiled EF- $1 \alpha$ sequences from the Fusarium-ID v2 database (1,651 sequences) (Geiser et al. 2004), GenBank type sequences (449 sequences), and 14 recent publications (626 sequences) (Supplementary Table S1) in order to represent the 27 species complexes and 4 species described by O'Donnell et al. (2012). These sequences spanned the taxonomic diversity in the genus Fusarium, except for the albidum and ventricosum species complexes (sequences were not available at the time of compilation). Sequences were aligned with MUSCLE (version 3.8.31) and trimmed to include only the region between the $3^{\prime}$ end of the first exon and the $5^{\prime}$ end of the third exon (Edgar 2004). This region was targeted to maximize the amount of intron sequence while limiting the amplicon size to approximately $450 \mathrm{bp}$ (Supplementary Fig. S1). Truncated $(n=291)$ and nonunique $(n=$ 1,716 ) sequences were removed manually and with the 'sequniq' function of GenomeTools (version 1.5.10) to yield a database of 718 Fusarium EF-1 $\alpha$ sequences (Supplementary Dataset S1) (Gremme et al. 2013). A neighbor-joining tree was built from this alignment in Geneious Prime version 2021.1.1 with the Tamura-Nei genetic distance model (Supplementary Datasets S2 and S3). Clades on this tree were manually assigned to 27 species complexes and 4 species proposed by O'Donnell et al. (2012) based on shared topology trees built on the $R P B 1 / R P B 2$ loci and previously reported species-level classifications of representative sequences. 
The second database comprised all sequences in NCBI GenBank matching the search terms "Fusarium translation elongation factor 1-alpha" ( $n=30,312)$ on 4 January 2021 . These sequences were aligned (by MUSCLE) and trimmed (in Geneious Prime) to include only the target amplicon region. Sequences were removed that did not contain the full target region $(n=8,184)$, contained ambiguous bases $(n=702)$, contained missense or nonsense mutations in the first or third exons $(n=69)$, or were "UNVERIFIED" $(n=198)$. The remaining 21,159 sequences were deduplicated to remove nonunique sequences ( $n=18,844$ removed). We observed that prospective primer binding sequences from the basal, $F$. dimerum species complex were highly divergent, and we chose not to target these species complexes in subsequent primer design. We removed $F$. dimerum species complex sequences to yield a final database of 2,284 sequences (Supplementary Dataset S4).

Template-specific primer design. We designed primers targeting the EF-1 $\alpha$ locus based on 718 unique sequences in the first database. A forward primer (called "fefF") was designed 2 nucleotides (nt) downstream of the "Fa" primer designed by Karlsson et al. (2016) to avoid sequence divergence in some $F$. solani isolates (Supplementary Fig. S1). The reverse primer (called "fefR") was designed to target the $5^{\prime}$ end of the third exon. Primer sequences were fefF: $5^{\prime}$-GTCATCGGCCACGTCGACTCTGG-3' and fefR: 5'-CCTTDCCGAGCTCRGCGGCTTCC-3', where D represents A, G or T, and R represents A or G.

Oligo design for two-step PCR library construction. Amplicon libraries can be produced with two sequential PCR amplification steps (called the "two-step" method). An advantage of this method is that unique, template-specific barcodes do not need to be purchased for every sample, because the same indices can be used for multiple amplicon targets (Supplementary Fig. S2). Oligos were designed for two-step PCR library construction with EF-1 $\alpha$ and ITS loci. The full oligos used in PCR amplification contained the Illumina Truseq adapter followed by a spacer ( 0 to 3 randomly selected nucleotides ["N"]), a 2-nt linker (not complementary to any nucleotides $5^{\prime}$ of the primer binding sites), and the template-specific primer sequence: fefF or fefR for EF-1 $\alpha$, and ITS4ngs (White et al. 1990) and ITS86f (Turenne et al. 1999) for ITS2 (Supplementary Dataset S5). The 0- to 3-nt spacers upstream of primer sequences were included to improve cluster diversity on the flow cell and subsequent sequence quality. Oligos of the four spacer types were pooled with equal molarity.

Oligo design ligation-based library construction after inlinebarcoded PCR amplification. Libraries can also be generated by ligating Illumina flow-cell adapters to amplicons produced with primers that have a sample-specific barcode ("inline-barcoded" primers) (Supplementary Fig. S2). An advantage of this method is that it may require fewer cycles of amplification and is convenient when the same locus will be targeted for amplification across multiple studies. For inline-barcoded PCR amplification, forward primer oligos were designed with an 8-nt barcode, a 2-nt linker (the same as above), and the $f e f F$ template-specific sequence (Supplementary Dataset S5). The 8-nt barcodes had a minimum Hamming distance of 4, equal GC ratios, and no homopolymers longer than 2 nt (generated by 'create.dnabarcodes' in R) (Buschmann and Bystrykh 2013).

EF-1 $\alpha$ two-step PCR amplification. The first-step reaction mixture for EF-1 $\alpha$ PCR amplification contained a total reaction volume of $25 \mu 1,1 \times$ concentration of OneTaq Master Mix (New England Biolabs), $0.2 \mu \mathrm{M}$ each primer oligo mixture (fefF and fefR with 0 - to 3-nt spacers), $20 \mu \mathrm{g}$ of bovine serum albumen, and $60 \mathrm{ng}$ of template DNA. The first-step EF- $1 \alpha$ PCR assays were run in a Bio-Rad T100 Thermal Cycler with these thermocycler parameters: $3 \mathrm{~min}$ at $95^{\circ} \mathrm{C}$; then, 30 cycles of $95^{\circ} \mathrm{C}$ for $30 \mathrm{~s}, 68^{\circ} \mathrm{C}$ for
$30 \mathrm{~s}$, and $72^{\circ} \mathrm{C}$ for $30 \mathrm{~s}$; followed by a final extension of $72^{\circ} \mathrm{C}$ for $5 \mathrm{~min}$. The high $\left(68^{\circ} \mathrm{C}\right)$ annealing temperature was selected from a gradient for increased specificity with optimal amplification (data not shown). The second-step PCR amplification was conducted by mixing $5 \mu l$ of step 1 reaction mixture (after thermocycling) with $1 \times$ OneTaq Master Mix and 0.24 $\mu \mathrm{M}$ unique dual-indexed TruSeq Illumina adapters (Supplementary Dataset S5). This mixture was run in the same thermocycler with the following settings: $95^{\circ} \mathrm{C}$ for $3 \mathrm{~min} ; 10$ cycles of $95^{\circ} \mathrm{C}$ for $30 \mathrm{~s}, 55^{\circ} \mathrm{C}$ for $30 \mathrm{~s}$, and $72^{\circ} \mathrm{C}$ for $30 \mathrm{~s}$; then, $72^{\circ} \mathrm{C}$ for $5 \mathrm{~min}$.

ITS2 two-step PCR amplification. Amplification of ITS2 was conducted with $1 \times$ concentration of OneTaq Master Mix; $0.2 \mu \mathrm{M}$ oligos containing TruSeq adapters, spacers, and either ITS4ngs or ITS86f template-specific sequences (Turenne et al. 1999; White et al. 1990); $20 \mu \mathrm{g}$ of bovine serum albumen; and $60 \mathrm{ng}$ of template DNA. The reaction was run in a Bio-Rad T100 Thermal Cycler with $95^{\circ} \mathrm{C}$ for $3 \mathrm{~min} ; 30$ cycles of $95^{\circ} \mathrm{C}$ for $30 \mathrm{~s}, 56^{\circ} \mathrm{C}$ for $15 \mathrm{~s}$, and $72^{\circ} \mathrm{C}$ for $30 \mathrm{~s}$; and $72^{\circ} \mathrm{C}$ for $1 \mathrm{~min}$. The second step PCR was conducted as described for the EF- $1 \alpha$ amplicons.

EF-1 $\alpha$ inline-barcoded PCR amplification. The reaction mixture $(25 \mu \mathrm{l})$ for inline-barcoded EF-1 $\alpha$ PCR amplification contained $1 \times$ concentration of DreamTaq Master Mix (Invitrogen), $0.2 \mu \mathrm{M}$ inline-barcoded fefF primer, $0.2 \mu \mathrm{M}$ template-specific fefR primer, $20 \mu \mathrm{g}$ of bovine serum albumen, and $60 \mathrm{ng}$ of template DNA. Reactions were run in a Bio-Rad T100 Thermal Cycler with 3 min at $95^{\circ} \mathrm{C} ; 35$ cycles of $95^{\circ} \mathrm{C}$ for $30 \mathrm{~s}, 68^{\circ} \mathrm{C}$ for $30 \mathrm{~s}$, and $72^{\circ} \mathrm{C}$ for $30 \mathrm{~s}$; and a final extension of $72^{\circ} \mathrm{C}$ for $5 \mathrm{~min}$.

Library preparation and high-throughput sequencing. We included a barcoded negative (water) control in every PCR (for ITS and $\mathrm{EF}-1 \alpha$ ), and these were included in all library preparations. PCR amplicons resulting from the two-step PCR amplification method were purified with AMPure XP beads (Beckman-Coulter) following the manufacturer's protocol. EF-1 $\alpha$ amplicons were size selected with BluePippin (Sage Science) for fragments between 350 and 600 bp. The EF- $1 \alpha$ and ITS2 libraries were then pooled and sequenced on an Illumina MiSeq with 300-nt paired-end reads.

Amplicons generated by the inline-barcoded method were pooled into a single mixture, then size selected to 350- to 550-bp fragments with the Zymo Research Gel Recovery kit (Zymo Research). Libraries were prepared for MiSeq sequencing with the ligation-based Kapa Hyper Prep library preparation kits at the DNA Technologies Core Facility (University of California-Davis). The resulting DNA was sequenced on an Illumina MiSeq with 300-nt paired-end reads.

Read processing and analysis. Reads were classified to the species $(E F-1 \alpha)$ or genus (ITS) level with multiple functions from the dbcAmplicons program (version 0.9.0; https://github.com/ msettles/dbcAmplicons) (Williams et al. 2017). We first used the 'preprocess' function with default parameters to identify and remove primer and barcode sequences. Paired-end reads were merged FLASH2 (version 2.2.00) via the 'join' function (maximum mismatch rate "-x" $=0.2$ for EF- $1 \alpha$ and 0.1 for ITS) (Magoc and Salzberg 2011). Joined EF-1 $\alpha$ reads were classified to species with 'classify', which uses the RDP naïve Bayesian classifier with our 718-sequence reference database and a minimum-length parameter ('minL') of $350 \mathrm{nt}$. Joined ITS reads were classified to genus-level phylotypes with the 'classify' function and the UNITE reference database. The read counts per taxonomic classification were summarized by the 'abundance' function.

To identify subspecies-level haplotypes for EF-1 $\alpha$ reads, we generated species-specific alignments for use with 'oligotyping' (version 2.1; https://merenlab.org/software/oligotyping/) (Eren et al. 2013). First, we extracted 'fastq'-formatted reads that corresponded to specific Fusarium spp. with the dbcAmplicons 'extract' function. Reads were converted to 'fasta' format with the bbmap 
function 'reformat.sh' and aligned against a template alignment with PyNAST (minimum percent identity of $80 \%$; version 0.1 ) (Caporaso et al. 2010). Template alignments were generated for each species complex by subsetting reads corresponding to the desired taxonomy (e.g., F. oxysporum species complex) from the comprehensive, 2,284-sequence database.

Shannon's entropy was then determined for each position of each alignment with the 'entropy-analysis' function. Sites that were known to be polymorphic (from the analysis of isolates cultured from these soils) or had high-entropy values were manually selected for oligotyping, and the 'oligotype' function was run with the following parameters: "-A 500 -M 150". The species-level relative abundances (determined by dbcAmplicons 'classify') were split into the relative proportions of their constituent oligotypes. This resulted in a relative abundance table in which all Fusarium ASVs were classified to the subspecies level.

Analysis of ITS and EF-1 $\alpha$ amplicon data. Representative sequences from ITS phylotypes and EF- $1 \alpha$ ASVs were aligned by MUSCLE and their phylogenetic distance was calculated by fasttree (Version 2.1.10). We used 'phyloseq' (version 1.32.0) in $\mathrm{R}$ (version 4.0.2) to import read counts, taxonomy, sample information, and phylogenetic distance (McMurdie and Holmes 2013; $\mathrm{R}$ Core Team 2013). Count data sets were normalized using the phyloseq 'transform_sample_counts' function by dividing each count in a sample by the total sample sum. Phylotypes or ASVs whose normalized total abundance was $>1 \%$ were identified and visualized via stacked bar plots using the phyloseq 'plot_bar' function. Nonmetric multidimensional scaling was calculated and visualized with the phyloseq 'ordination' (method $=$ NMDS, distance $=$ wunifrac) and 'plot_ordination' functions, respectively.

The phyloseq function 'phyloseq_to_deseq2' was utilized to define a DESeq2 object containing an explanatory variable formula "group", which was generated by combining sample data variables "treatment type" and "extraction location" (design $=\sim$ group). To account for samples containing 0 counts, the DESeq 2 function 'estimateSizeFactors' was used (version 1.28.1; type = poscounts) (Love et al. 2014). The DESeq2 function 'DESeq2' then was used to perform comparisons using nonnormalized count data (test $=$ wald, fitType $=$ mean).

Microbiome objects with normalized counts were created using the microbiome (version 1.4.2) 'transform' function (transform = compositional) (Lahti and Shetty 2017). The 'adonis' function from vegan (version 2.5-7) was then used to perform permutational multivariate analysis of variance (PERMANOVA) on a weighted distance matrix created using the phyloseq 'distance' function (method = wunifrac). Codes used for $\mathrm{R}$ analysis of microbiome data in this study are available at http://www.github.com/pmhenry/ USDA-ARS-fusarium-community.

Performance on artificial communities. To evaluate our EF- $1 \alpha$ amplicon sequencing method, we mixed DNA from eight Fusarium isolates in five different proportions (called 'mock communities') and compared the observed "post-PCR" percentage of reads of each isolate with the expected percentages of template DNA ("pre-PCR") in each mock community. Post-PCR percentages for the eight isolates were obtained for the two library preparation methods that we tested (i.e., inline-barcoded and two-step PCR); thus, for each isolate there were 2 observed versus expected comparisons (16 total).

We determined whether the variability in measurements of DNA concentration could account for differences in observed versus expected relative abundances of each isolate. DNA concentration was quantified three times for each isolate by Qubit (version 2.0), and these estimates were averaged to determine the mean pre-PCR DNA concentration per isolate. From the three measurements of each isolate's input DNA concentration, we generated a normal distribution of DNA concentrations ( $\mathrm{R}$ package = 'stats'; function = 'rnorm'). These distributions were sampled 10,000 independent times for the concentrations (in counts per thousand [cpt]) of the eight isolates ( $\mathrm{R}$ package $=$ 'stats'; function $=$ 'rnorm'). Then, we subtracted the observed cpt (from amplicon sequence analysis) from the simulated cpt (based on a normal distribution) for each PCR replicate. The $P$ values are the fraction of the two-sided Wilcoxon signed rank test in which the post-PCR minus simulated pre-PCR values were not significantly $(\alpha=0.05)$ different from zero after adjustment for 16 comparisons with the false discovery rate $(\mathrm{R}$ package $=$ 'stats'; function = 'wilcox.test','p.adjust(method="BH”) (https:// github.com/objetora/lepstein/blob/master/pcriw.r) (Benjamini and Hochberg 1995).

We used the genome size of each isolate to estimate the expected numbers of single-copy EF- $1 \alpha$ template sequences per nanogram of DNA. The genome sizes were known for two isolates: GL1080 (Henry et al. 2021) and FolCA3 (Henry et al. 2019b). The genome size of the F. oxysporum f. sp. apii race 2 isolate Foa 40 was based on the size of F. oxysporum f. sp. apii race 2 strain 207A (Henry et al. 2020b); race 2 is monomorphic in California (Epstein et al. 2017). For the remaining isolates, we used the mean size of GenBank assemblies corresponding to the species complex (Supplementary Table S2).

Culture-dependent characterization of Fusarium communities from field soil before infestation with Fof. Approximately 300 liters of soil from a depth of 0 to $15 \mathrm{~cm}$ was collected near Watsonville, CA from an organic strawberry field that did not have a history of Fusarium wilt of strawberry. This soil was passed through a 4-mm mesh sieve to remove rocks and plant debris and homogenized by mixing for $1 \mathrm{~h}$ in a potting soil mixer. Three 5-g subsamples were taken from the bulk mixture, mixed vigorously with $1 \%$ sodium hexametaphosphate, diluted with $0.1 \%$ agar solution, and plated on Komada's medium with a detection limit of $50 \mathrm{CFU} / \mathrm{g}$. All colonies with aerial mycelia that was not green (to avoid Trichoderma and Penicillium spp.) were single-hyphal tipped (with sterile scalpels under a dissecting scope) to obtain pure cultures. DNA was extracted from each culture with the OmniGen-X Purespin gDNA extraction kit and the EF-1 $\alpha$ locus amplified with EF1 and EF2 primers, as described by Henry et al. (2021). Amplicons were purified with the Bioneer Accuprep PCR purification kit and sequenced from both ends on an ABI 3130XL Capillary Electrophoresis Genetic Analyzer. We used Geneious Prime to analyze reads $(2 \times$ coverage for each sample) and generate consensus sequences from the fefF and fefR amplicon regions. Unique sequence types were quantified by the 'fastx_collapser' tool in the fastx_toolkit (version 0.0.13).

Inoculum and soil preparation. A hygromycin-resistant transformant of a highly virulent strain of Fof was previously generated by Agrobacterium-mediated transformation (Henry et al. 2020a). Eighteen 100-mm-diameter Petri dishes of potato dextrose agar were inoculated with this strain (called GL1080 ${ }^{\text {hyg }}{ }^{\text {) }}$ ) and grown for 10 days at $25^{\circ} \mathrm{C}$ under continuous fluorescent lighting. Infested agar was then blended in sterile deionized water for 2 min and evenly hand mixed into 10 liters of sterile sand. This infested sand mixture was stirred manually daily for 1 week until completely dry. Infested sand was mixed thoroughly with 85 liters of the field soil described above. This mixture is hereafter referred to as "Fof-infested soil".

Plant growth, incorporation, and incubation. Ten-day old seedlings of five crop treatments (broccoli, spinach, lettuce, raspberry, and blackberry) as well as strawberry crowns that had broken dormancy (from Fof-susceptible and -resistant cultivars) were transplanted into 2-liter pots containing Fof-infested soil $(n=6$ per treatment) and transferred to a controlled-environment facility with a 12-h photoperiod and temperatures of $25^{\circ} \mathrm{C}$ (day) and $18^{\circ} \mathrm{C}$ (night) 
(Table 1). Six pots filled with Fof-infested soil were left unplanted as a "no-plant" control. The six replicate pots/treatment were arranged in a completely randomized design. Pots were checked daily for 6 weeks for weeds, and irrigated with a nutrient solution containing $\mathrm{NH}_{4}-\mathrm{N}$ at $5.99 \mathrm{mg} / \mathrm{liter}, \mathrm{NO}_{3}-\mathrm{N}$ at $70.39 \mathrm{mg} / \mathrm{liter}, \mathrm{P}$ at 53.92 $\mathrm{mg} / \mathrm{liter}, \mathrm{K}$ at $113.83 \mathrm{mg} / \mathrm{liter}, \mathrm{Ca}$ at $170.75 \mathrm{mg} / \mathrm{liter}, \mathrm{Mg}$ at 29.35 $\mathrm{mg} / \mathrm{liter}$, and $\mathrm{S}$ at $38.64 \mathrm{mg} / \mathrm{liter}$. After this period, aboveground plant biomass was collected and weighed, and a sample of feeder roots $(<1 \mathrm{~mm}$ in diameter) was saved for later analysis. Soil was removed from the pot and thoroughly mixed, and an approximately 200 -g subsample was saved. Plant biomass was then cut into approximately $1-\mathrm{cm}$ fragments and mixed evenly with the soil, which was then returned to the pot. For the no-plant treatment, soil was removed, mixed, sampled, and returned to the pot. Pots were then returned to the growth chamber for a 6-week fallow period, in which all treatments were irrigated weekly with $200 \mathrm{ml}$ of water. Then, soil was sampled and pots were replanted with the crop that had previously been grown. The entire process of plant growth (6 weeks), fragmentation and incorporation, and fallowing (6 weeks) was conducted twice, so that each pot was subjected to two plant growth and incubation periods of the same crop (Fig. 1A). All pots were thereafter irrigated weekly with $200 \mathrm{ml}$ of water for 28 weeks, at which point soils were sampled for a final time. At this final timepoint, soils were passed through a 4-mm mesh sieve to remove plant debris. This experiment was conducted three times.

Soil sampling, culture-dependent quantification of Fof, and DNA extraction. Soil was sampled as described above immediately after infesting with Fof at the beginning of the experiment, and then at $6,12,18,24$, and 52 weeks postinitiation (wpi) of the experiment (Fig. 1A). At each timepoint, soil was stored at $4^{\circ} \mathrm{C}$ for no longer than 1 week prior to dilution plating on Komada's medium containing hygromycin B at $100 \mathrm{mg} / \mathrm{liter}$ (Komada 1975). Few other microorganisms grow on this medium, and colonies morphologically identifiable as Fof were enumerated after 5 to 7 days of incubation at $23^{\circ} \mathrm{C}$ under continuous fluorescent lighting. Two assays were conducted per pot and the average of these values was used in subsequent statistical analyses. Soil for DNA extraction was collected from all pots ( $n=6$ per treatment) at 52 wpi and stored at $-80^{\circ} \mathrm{C}$ until the time of extraction. DNA was extracted from 500-mg soil samples with the Zymo Research Quick-DNA Fecal/Soil extraction kit as per the manufacturer's instructions. PCR and library preparation were as described for the two-step method.

Fine root tissue sampling and DNA extraction. Feeder roots were submerged in sterile $1 \%$ sodium hexametaphosphate solution

TABLE 1

Rotation crop treatments in the Fusarium oxysporum f. sp. fragariae experiment

\begin{tabular}{ll}
\hline Crop/treatment & \multicolumn{1}{c}{ Cultivar } \\
\hline Broccoli & Marathon F1 \\
\hline Spinach & Whale \\
\hline Lettuce & Green Towers \\
\hline Raspberry & Heritage \\
\hline Blackberry & Black satin \\
\hline Strawberry (resistant) & Ventana \\
\hline Strawberry (susceptible) & Sweet Anne \\
\hline No plant (irrigated) & a \\
\hline $\begin{array}{l}\text { a This treatment was irrigated when dry (approximately once per } \\
\text { week) for the duration of the experiment. }\end{array}$
\end{tabular}

(to disperse soil aggregates) and shaken at $100 \mathrm{rpm}$ for $10 \mathrm{~min}$ (Kettler et al. 2001). Tissue was transferred to a tube with fresh, sterile $1 \%$ sodium hexametaphosphate solution, shaken again, and sonicated in a Branson 5510 water bath (Marshall Scientific, LLC) for $7 \mathrm{~min}$. Tissue was then transferred to a tube with sterile, deionized water and shaken for $10 \mathrm{~min}$ at $100 \mathrm{rpm}$. Root tissues were then transferred to sterile 2-ml centrifuge tubes, flash frozen in liquid nitrogen, and lyophilized prior to DNA extraction. A modified cetyltrimethylammonium bromide extraction protocol was used to isolate DNA from the plant tissue (Brandfass and Karlovsky 2008). The two-step PCR and library preparation method was used for these DNA samples.

Impact of an 18-year cover crop and conservation tillage study on Fusarium communities. We investigated the impact of cover cropping and conservation tillage on Fusarium communities at the long-term (18-year) Conservation Agriculture Systems Innovation site at the University of California's West Side Research and Extension Center in Five Points, CA $\left(36^{\circ} 20^{\prime} 29^{\prime \prime} \mathrm{N}, 120^{\circ} 7^{\prime} 14^{\prime \prime} \mathrm{W}\right)$ (Mitchell et al. 2021). The field has Panoche clay loam with two experiments that each contain four replicated blocks of four management treatments: (i) standard tillage with no off-season cover crop, (ii) standard tillage with an off-season cover crop, (iii) conservation tillage with no off-season cover crop, and (iv) conservation

A Soil: EF1 - culture dependent

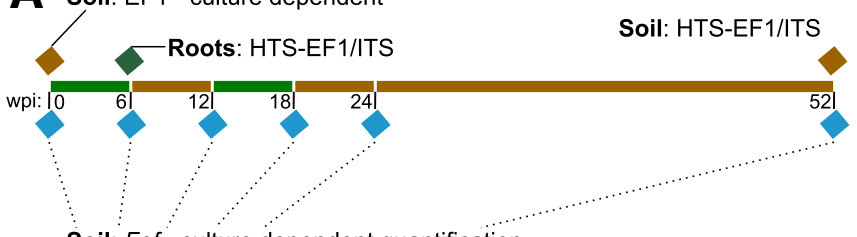

Soil: Fof - culture dependent quantification

$\begin{array}{ll}\text { Treatments: } & \text { Raspberry (RB) } \\ \text { Blackberry (BB) } & \text { Strawberry-susc. (SW) } \\ \text { Broccoli (BR) } & \text { Strawberry-res. (VT) } \\ \text { Lettuce (LE) } & \text { No plant control (NP) }\end{array}$

B

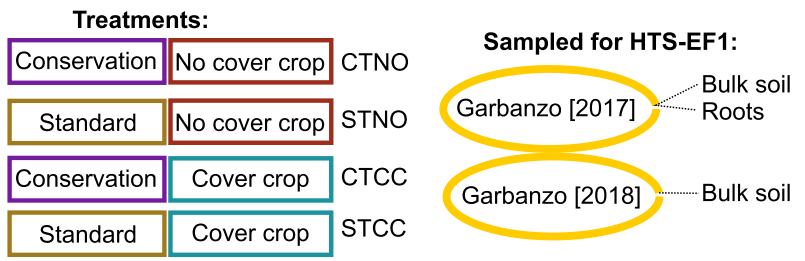

Fig. 1. Experimental design for simulated crop rotation experiments and 18-year conservation tillage and cover cropping trials. A, Depiction of the timing for data collected from the simulated crop rotation experiments. Plants were grown between 0 and 6 weeks postinitiation (wpi) of the experiment in soil infested with Fusarium oxysporum f. sp. fragariae (Fof), then fragmented and mixed into the soil. The soil was then irrigated weekly between 6 and 12 wpi as plant tissues decomposed. Pots were replanted at 12 wpi fragmented and tilled into the soil at 18 wpi, and then irrigated weekly until 52 wpi. Green intervals depict time periods when plants were growing ( 0 to 6 and 12 to $18 \mathrm{wpi}$ ) and brown intervals depict time periods when soil was incubated and tissues were decomposing. The amount of Fof in soil was quantified by plating on selective medium at $0,6,12,18,24$, and 52 wpi. DNA was extracted from roots from 6 wpi and soil from 52 wpi for high-throughput sequencing (HTS) of translation elongation factor $1 \alpha(\mathrm{EF}-1 \alpha)$ and internal transcribed spacer (ITS) amplicons. B, Visualization of treatments and sampling procedures after 18-year tillage and cover-cropping field trials. Treatments included conservation tillage or standard tillage and cover cropped or not. Garbanzo bean plants were grown in the winter of 2017 and 2018 and, during these years, bulk soil was sampled for HTS of EF-1 $\alpha$ amplicons. DNA was also extracted from roots in 2017 for HTS of EF-1 $\alpha$ amplicons. 
tillage with an off-season cover crop (Fig. 1B). Each plot was $10 \mathrm{~m}$ wide by $100 \mathrm{~m}$ long with a $10-\mathrm{m}$ buffer between plots. From 2014 through 2018, there was a 2-year crop rotation alternating between garbanzo bean (cultivar UC27) and sorghum; four blocks were planted to each crop in each year. The cover-crop-treated plots had an annual fall planting, starting in 2014, with a mix of triticale, cereal rye, common vetch, radish, and clover.

On 9 May 2017 and 12 June 2018, 10 random bulk soil samples (0 to $15 \mathrm{~cm}$ deep, $13 \mathrm{~cm}$ from a garbanzo [Cicer arietinum] stem) per plot were pooled, mixed, air dried, and mixed again. In 2017, the fine fibrous roots from 10 random garbanzo bean plants were also collected, washed in water, sonicated in a Branson 5510 water bath, blotted dry, stored at $-70^{\circ} \mathrm{C}$ until lyophilization, then ground in liquid $\mathrm{N}_{2}$ with a mortar and pestle. The DNA from 50-mg root samples was extracted with ZR Soil Microbe DNA Miniprep kits. PCR and library preparation were as described for the inline-barcoded method for 2017 samples and by the two-step amplification method for 2018 samples.

Data availability. Amplicon sequences were deposited in the GenBank Sequence Read Archive under accession codes SRR17775566 and SRR17775565. All data will be made available upon request.

\section{RESULTS}

Primer design. To design and evaluate the compatibility of new primers for Fusarium EF-1 $\alpha$ amplicon sequencing, we developed a database of 21,159 high-quality Fusarium EF-1 $\alpha$ sequences from GenBank, of which 2,284 were unique. The fefF primer was identical to its binding region in $97.5 \%$ of the 2,284 sequences; 58 sequences $(2.5 \%)$ had silent mutations in this region. The fefR primer contained two degenerate bases, which made it perfectly complementary to $99.3 \%$ of sequences; there were 2 sequences with silent mutations and 14 sequences with missense mutations. Mismatches on either fefF or fefR were not clustered within the same species complex and were dispersed, apparently at random, across seven species complexes (Supplementary Table S3), suggesting that they are sequencing errors rather than true polymorphisms.

Quantification of Fusarium ASVs from mock communities. We identified each input isolate from amplicon sequences by oligotyping, and this method reconstructed the expected EF-1 $\alpha$ sequences with $100 \%$ sequence identity. Because the differences between post- and pre-PCR values may have been due to inaccuracies in the estimates of the pre-PCR values, we sampled simulated

TABLE 2

Difference between observed and expected relative abundances of eight Fusarium isolates in five mock community mixtures ${ }^{a}$

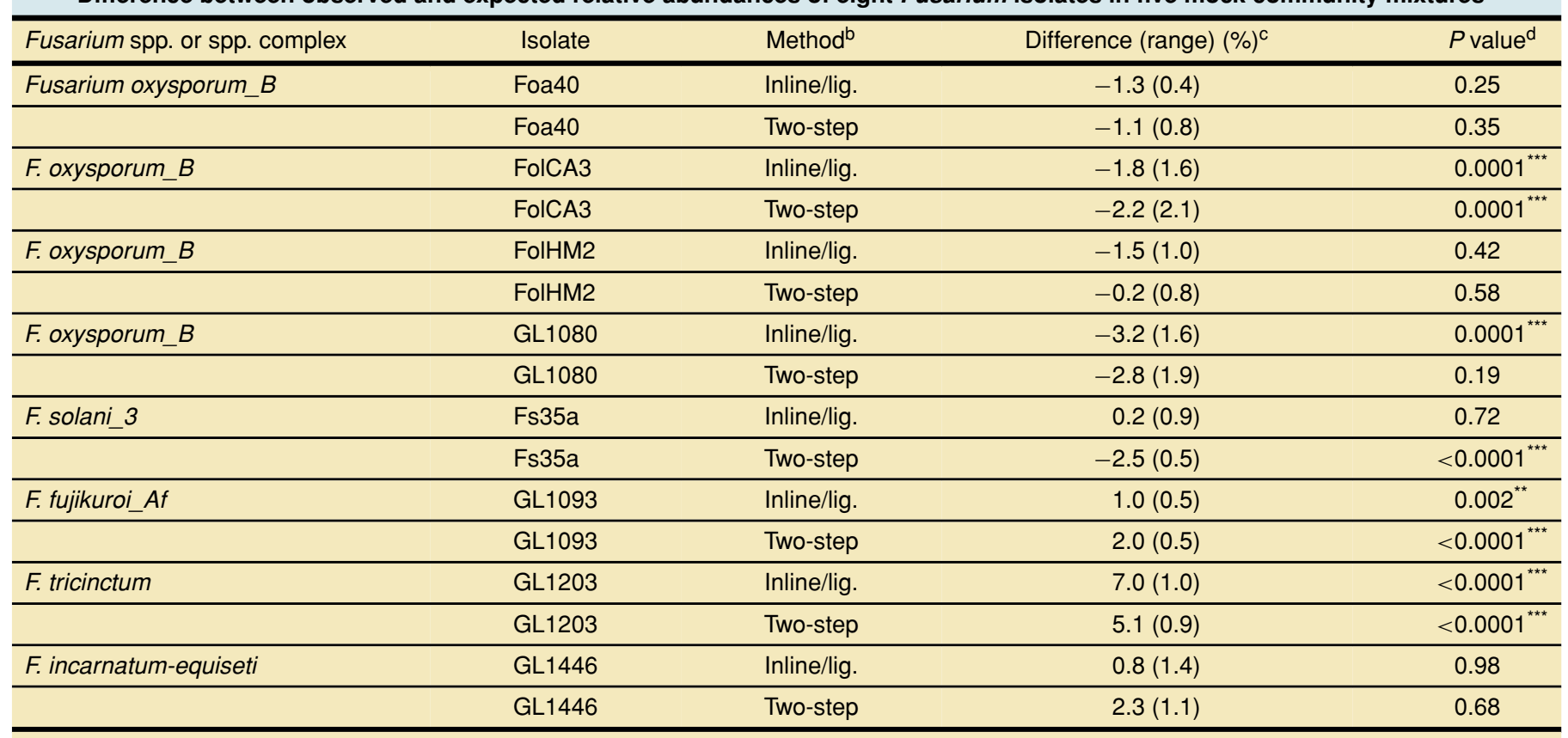

a Each sample contained eight Fusarium spp. isolates in one of five different proportions.

b The PCR and library preparation method was either with inline barcodes followed by ligation (Inline/lig.) or by two-step PCR amplification (Two-step). In the inline/lig. method, a sample-specific 8-nucleotide (nt) barcode was attached to the template specific fefF primer during PCR. Amplicons were then pooled and prepared for Illumina sequencing with a ligation-based library preparation method. For the two-step PCR library preparation method, the fefF and fefR primers each had a $5^{\prime}$ Illumina TruSeq adapter sequence in the first round of amplification. The primers in the second round of amplification had the P5 or P7 Illumina adapters and an 8-nt sample-specific barcode. After the second round of amplification, amplicons were pooled and size selected before sequencing.

${ }^{c}$ Median percent difference (and interquartile range) between observed and expected. Pre-PCR estimates of quantity of each isolate were based on the measured DNA concentration of each isolate stock and their genome sizes, in order to estimate the number of single-copy translation elongation factor $1 \alpha$ templates of each isolate in each mix. For each isolate, PCR method, and PCR replicate, the percent difference was calculated as (post-PCR - mean pre-PCR) $\times 100$. Because the percent differences were not normally distributed, the column has the median values ( $n=15$ and 9 for the 2017 and 2018 PCR methods, respectively).

d $P$ value that pre-PCR variability could account for a significant difference between post- and pre-PCR. The three measurements of pre-PCR DNA concentration of each isolate were normally distributed, except for FolCA3. To test whether the post-PCR relative differences could be explained by the pre-PCR variability, we sampled 10,000 sets of eight values, one for each isolate, from the corresponding pre-PCR distributions that were based on the three measurements of DNA concentration for each isolate. The $P$ values are the fraction of the 10,000 samples that had a nonsignificant $(\alpha=0.05)$, two-sided Wilcoxon signed rank test adjusted for 16 comparisons with the false discovery rate $(\alpha=0.05)$ for the post-PCR - simulated pre-PCR values. 
estimates from the distribution of possible values. In 8 of the 16 cases, the relative difference was significant and the variability of the pre-PCR value could not account for the observed difference between the post-PCR and pre-PCR values (Table 2; Supplementary Fig. S3). Overall, PCR amplification did not affect the post-PCR percentage of Foa40, FolHM2, and GL1446 in the mixes (Table 2). With both methods, we observed bias for three isolates: an increase in the percentage of GL1093 (median $=1.5 \%$ ) and GL1203 (median $=6.0 \%)$ and a decrease in the percentage of FolCA3 $($ median $=-2.0 \%)($ Table 2$)$.
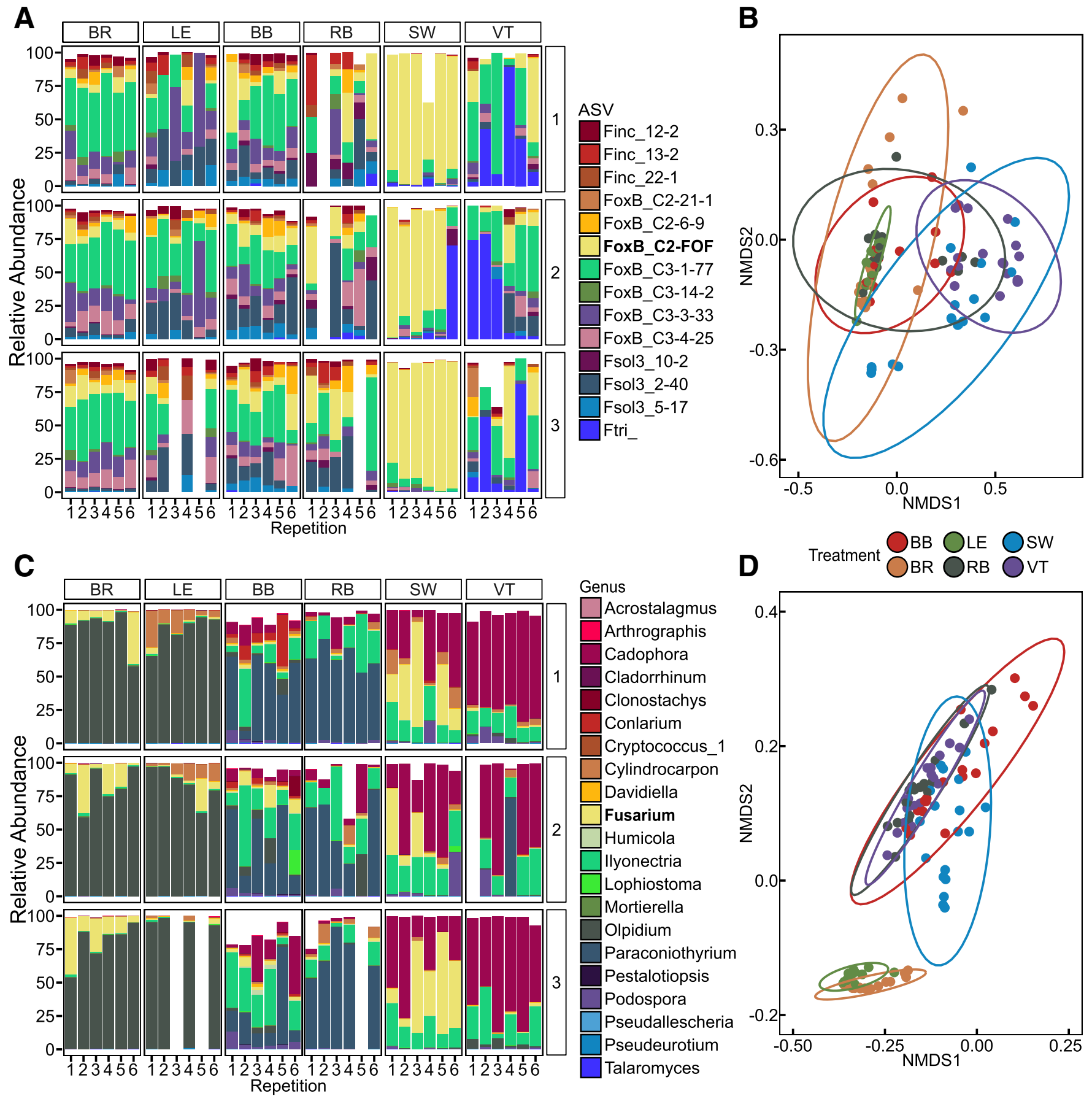

Fig. 2. Fusarium and fungal communities on the roots of six crop species harvested after 6 weeks of growth in Fusarium oxysporum f. sp. fragariae (Fof)-infested soil. Treatment codes are as follows: $\mathrm{BB}=$ blackberry, $\mathrm{BR}=$ broccoli, $\mathrm{LE}=$ lettuce, $\mathrm{RB}=$ raspberry, $\mathrm{SW}=$ Fof-susceptible strawberry cultivar 'Sweet Ann', and VT = Fof-resistant strawberry cultivar 'Ventana'. A, Stacked bar chart depicting the relative abundance of the top 14 most abundant Fusarium amplicon sequence variants (ASVs), based on translation elongation factor $1 \alpha$ (EF-1 $1 \alpha$ ). The ASV corresponding to the introduced strain of Fof is depicted in yellow and called FoxB_C2-FOF in the legend. ASV IDs starting with "Finc" are from the F. incarnatum-equiseti species complex, "FoxB" = F. oxysporum species complex, "Fsol3" = F. solani species complex 3, and "Ftri" = F. tricinctum species complex. Missing data were caused by sample loss during DNA extraction or a lack of target amplification. B, Nonmetric multidimensional scaling plot of Fusarium communities in roots based on EF-1 $\alpha$ amplicon analysis. C, Stacked bar chart depicting the relative abundance of the 21 most abundant fungal genera identified by internal transcribed spacer 2 (ITS2) amplicon sequence analysis. D, Nonmetric multidimensional scaling plot of fungal communities in roots based on ITS2 amplicon sequences. 
Fusarium community profiles reveal evidence for host preferences in both symptomatic and asymptomatic interactions. The profound niche advantage granted by host-specific pathogenicity was confirmed by our analysis of Fof relative abundance on root tissues of strawberry and other crop species growing in artificially Fof-infested soils. As revealed by EF- $1 \alpha$ amplicon sequencing, the Fusarium community on roots of the susceptible strawberry cultivar was dominated by the introduced Fof (isolate GL1080 ${ }^{\text {hyg }}$; ASV FoxB_C2-FOF), which had $\log _{2}$ fold-change $\left(\log _{2} \mathrm{FC}\right)>$ 6.17 differential abundance compared with any other treatment $(P<0.001)$ (Fig. 2; Supplementary Dataset S6). Concurrently, many $F$. incarnatum-equiseti, $F$. solani 3 , and F. oxysporum ASVs were at lower relative abundance in the susceptible strawberry treatment compared with the roots of other crops (Fig. 2; Supplementary Dataset S6). In the ITS data, the Fusarium genus was 1.59 to 6.53 $\log _{2} \mathrm{FC}$ more abundant on the susceptible strawberry roots than the roots of any other crop $(P<0.001)$ (Fig. 2).

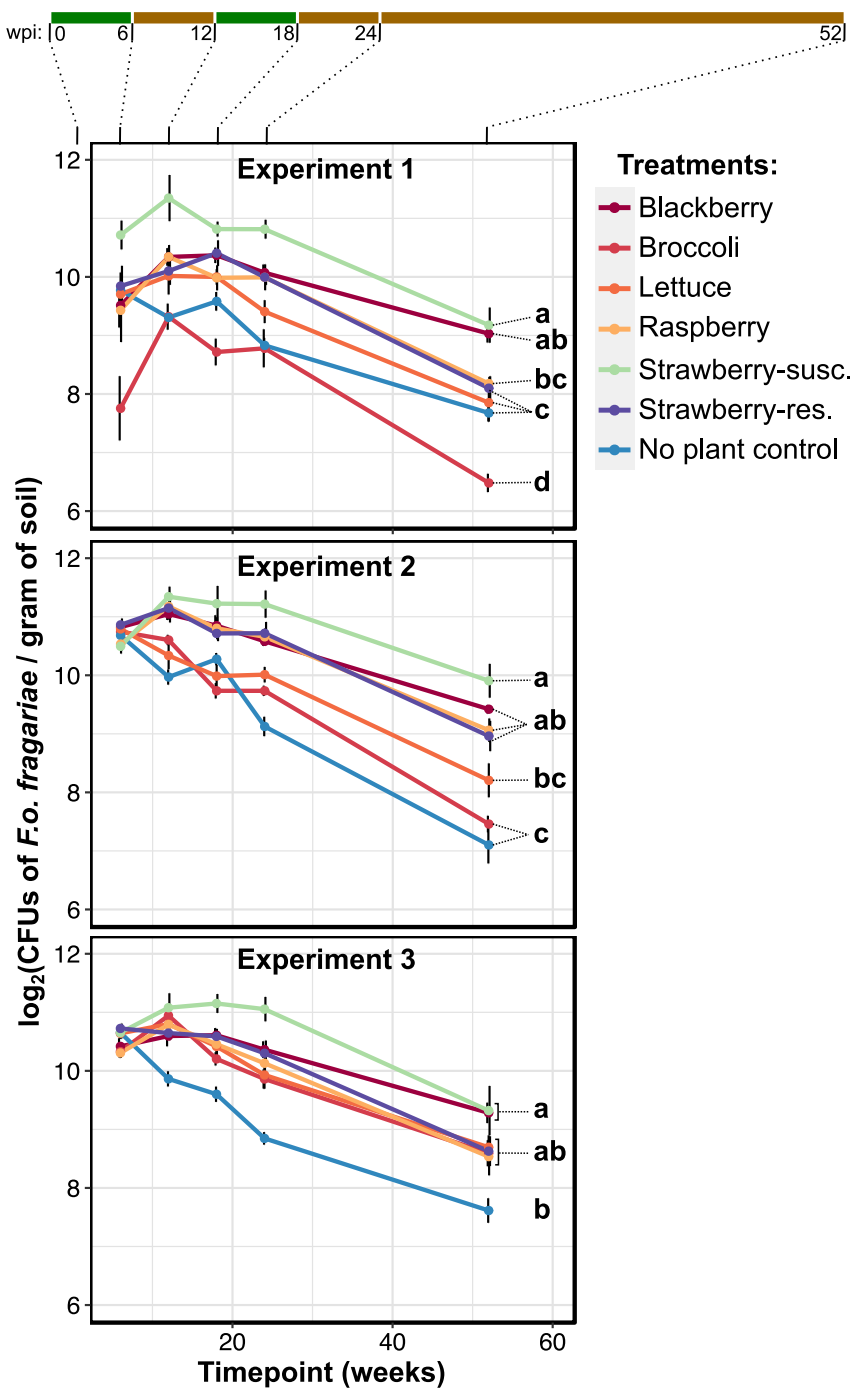

Fig. 3. Culture-based changes in the population density of Fusarium oxysporum f. sp. fragariae ( Fof; isolate $\mathrm{GL} 1080^{\text {hyg }}+$ ) after introduction into the soil of simulated crop rotations. $\log _{2}$ soil population densities of GL1080hyg+ at $6,12,18,24$, and 52 weeks postinitiation (wpi) after crop rotation treatments. Points show the mean at each timepoint, and error bars depict \pm 1 standard error. Tukey's honestly significant difference test was conducted on values from $52 \mathrm{wpi}$; the same letters adjacent to this timepoint are not significantly different $(\alpha=0.05)$. Starting inoculum density at 0 wpi was approximately $\log _{2}(16.6)$, or $1 \times 10^{6} \mathrm{CFU} / \mathrm{g}$.
Differential abundance analysis with DESeq2 also provided evidence that host preferences during asymptomatic root infections are common for Fusarium spp.; from these data, we observed many crop-dependent differential abundances of Fusarium ASVs on healthy root tissues. For example, Fof ASV FoxB_C2-FOF was at lower relative abundance in lettuce roots than blackberry or Fofresistant strawberry roots $\left(\log _{2} \mathrm{FC}=-1.76\right.$ and -1.73 , respectively; $P<0.03$ ) (Supplementary Dataset S6). In addition, the putatively nonpathogenic strain ASV FoxB_C3.3.33 was at higher relative abundance on broccoli than lettuce and raspberry $\left(\log _{2} \mathrm{FC}=\right.$ 2.21 and 1.99, respectively; $P<0.001$ ) (Supplementary Dataset S6). The most abundant ASV on most crops, FoxB_C3.1.77, was at higher relative abundance on broccoli roots compared with raspberry roots $\left(\log _{2} \mathrm{FC}=2.06 ; P<0.001\right)$ (Fig. 2; Supplementary Dataset S6). These differences suggest that crop rotation could augment the relative abundance of nonpathogenic strains that could compete with host-specific pathogens.

Soil inoculum densities of $\boldsymbol{F}$ of after repeated crop-fallow cycles and comparison with Fof ASV relative abundances. To compare the culture-independent data with culture-dependent counts of Fof, the soil inoculum densities of the introduced hygromycin-resistant Fof isolate GL1080 ${ }^{\text {hyg }}+$ were determined by soil dilution plating at five timepoints. The approximate inoculum density of GL1080 ${ }^{\text {hyg }}+$ in the initial soil mixture was $1 \times 10^{6} \mathrm{CFU} / \mathrm{g}$ of soil. Mean soil densities of GL1080 ${ }^{\text {hyg }}+$ were numerically highest in the susceptible strawberry treatment at all timepoints and experiments of all treatments (Fig. 3). Inoculum densities of GL1080 $0^{\text {hyg }}$ declined between the time of the second tillage at 12 wpi of the experiment and the final timepoint at $52 \mathrm{wpi}$. Two-way mixed-model analyses of variance (ANOVAs) at 52 wpi showed a significant effect of random effects (experiment and experiment-treatment interactions; $P=0.049$ and 0.005 , respectively). Therefore, data were analyzed separately for each experiment; the fixed effect of treatment was always significant $(P<0.001)$ in one-way ANOVAs (Fig. 3).

Overall, the relative abundance of the GL1080 ${ }^{\text {hyg }}+$ Fof ASV (FoxB_C2-FOF, as determined by amplicon sequencing) was not a good predictor of the abundance of Fof CFU/g of soil (determined by culture-dependent soil dilution plating). The only statistically

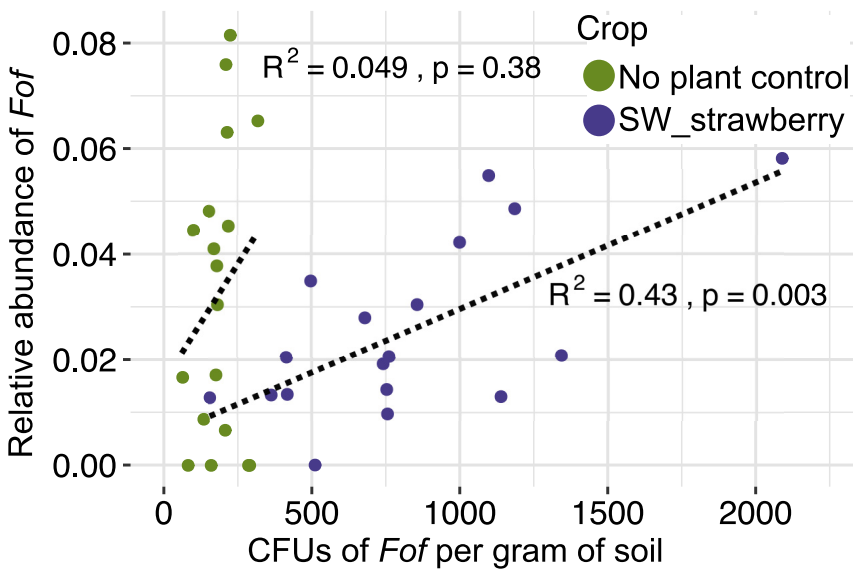

Fig. 4. Relationship between relative abundance and CFU per gram of soil of Fusarium oxysporum f. sp. fragariae (Fof) at 52 weeks postinitiation. Each point depicts the proportion of reads (i.e., relative abundance) corresponding to the Fof haplotype (y-axis) and the CFU of Fof per gram of soil (x-axis) for a single replicate. The two-step library preparation was used to generate reads for calculating Fof relative abundance. Two treatments are shown: the susceptible strawberry and fallow-irrigated treatments. 
significant correlations between Fof relative and actual abundance were observed in the susceptible strawberry $\left(R^{2}=0.43 ; P=0.003\right)$ and resistant strawberry $\left(R^{2}=0.23 ; P=0.043\right)$ (Fig. 4; Supplementary Fig. S4). No correlation between these variables was observed in the other treatments $\left(R^{2} \leq 0.068 ; P \geq 0.30\right)$ (Fig. 4; Supple- mentary Fig. S4). Furthermore, the Fof ASV FoxB_C2-FOF was not differentially abundant between the susceptible strawberry and no-plant treatments by DESeq 2 analysis in soil at 52 wpi (Supplementary Dataset S6). This result contrasts with culture-dependent quantifications of Fof from soil at that timepoint; the CFU of Fof
A

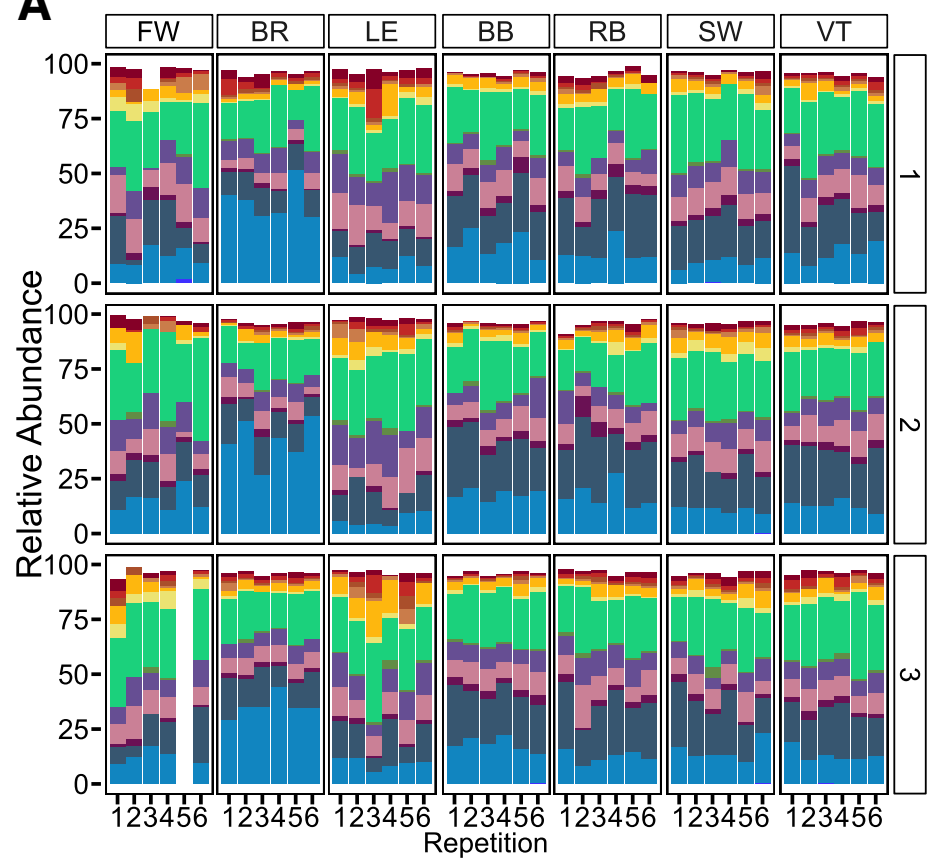

C

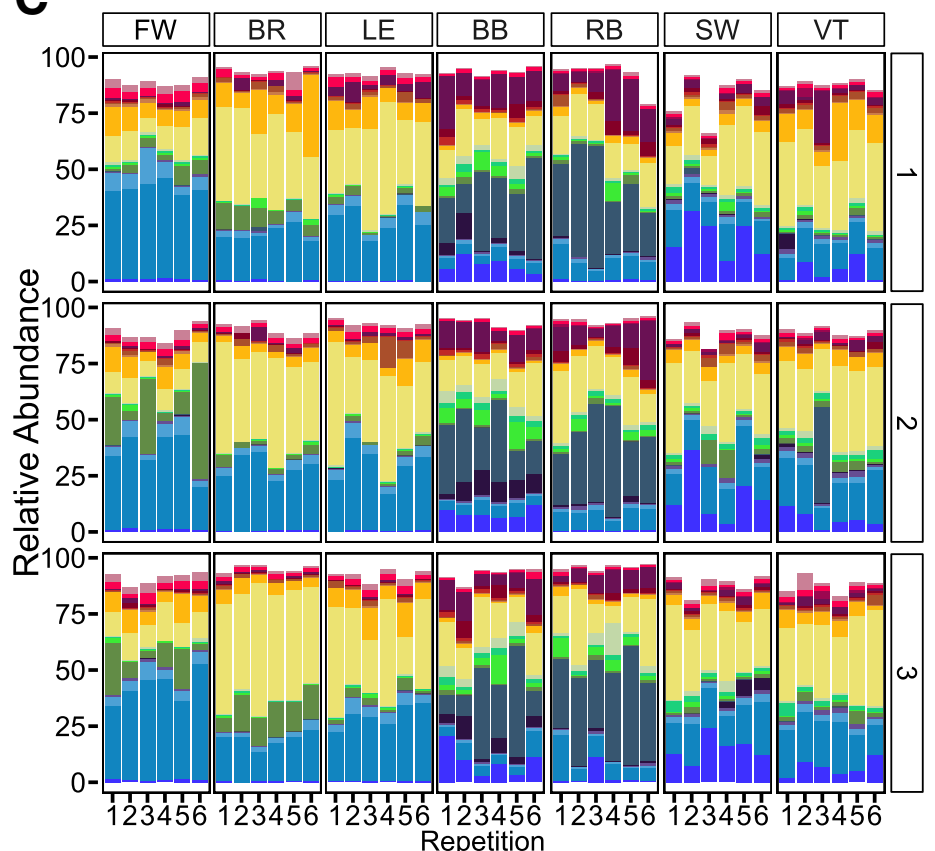

\section{ASV ID}

Finc_12-2

Finc_13-2

Finc_22-1

FoxB_C2-21-1

FoxB_C2-6-9

FoxB_C2-FOF

FoxB_C3-1-77

FoxB_C3-14-2

FoxB_C3-3-33

FoxB_C3-4-25

Fsol3_10-2

Fsol3_2-40

Fsol3_5-17

Ftri_

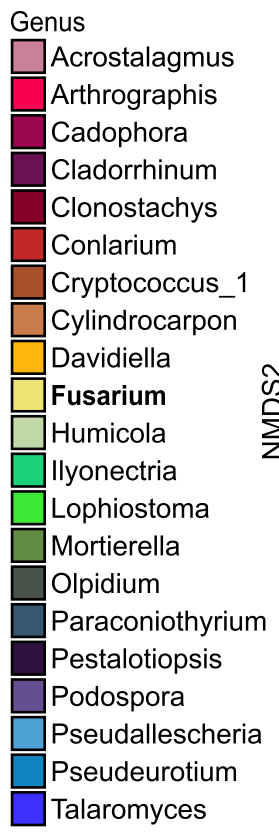

B

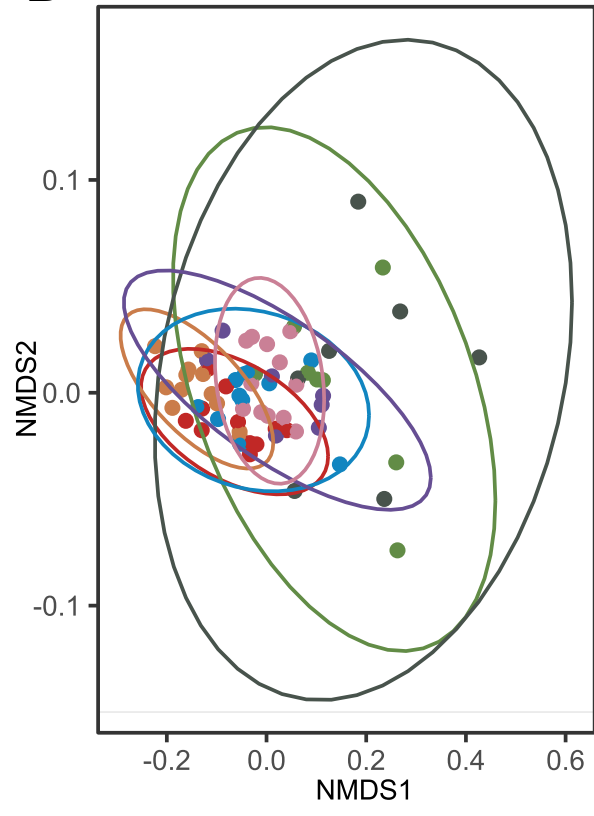

\section{D treatment ObB Ole Osw Ofw}

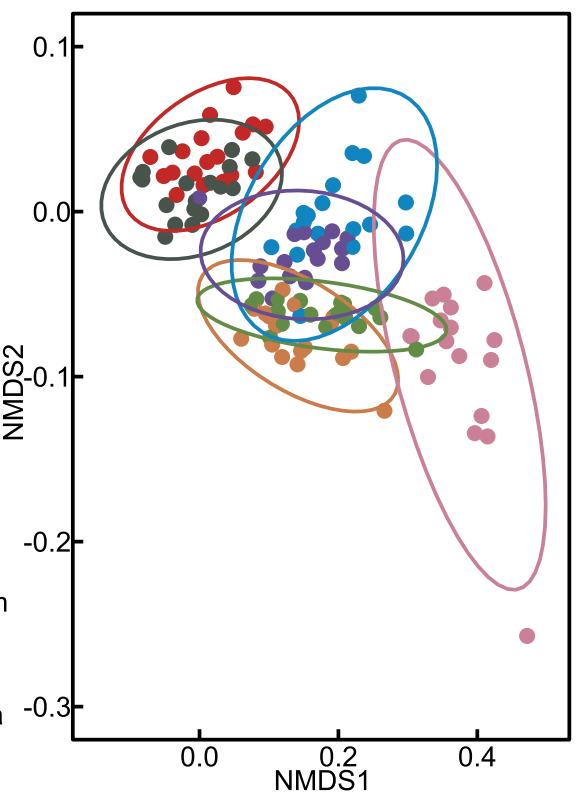

Fig. 5. Fusarium and fungal communities from soil after decomposition of six crop species and a fallow control at 52 weeks postinitiation (wpi) of the experiment. Treatment codes are as follows: $\mathrm{BB}=$ blackberry, $\mathrm{BR}=$ broccoli, $\mathrm{FW}=$ no-plant control, $\mathrm{LE}=$ lettuce, $\mathrm{RB}=$ raspberry, $\mathrm{SW}=$ Fusarium oxysporum f. sp. fragariae (Fof)-susceptible strawberry cultivar 'Sweet Ann', and VT = Fof-resistant strawberry cultivar 'Ventana'. A, Stacked bar chart depicting the relative abundance of the top 14 most abundant Fusarium amplicon sequence variants (ASVs) in soil at 52 wpi, based on translation elongation factor $1 \alpha(E F-1 \alpha)$ amplicon sequencing. The ASV corresponding to the introduced strain of Fof is depicted with yellow and called FoxB_C2-FOF in the legend. ASV IDs starting with "Finc" are from the $F$. incarnatum-equiseti species complex, "FoxB" = F. oxysporum species complex, "Fsol3" = F. solani species complex 3, and "Ftri" =F. tricinctum species complex. B, Nonmetric multidimensional scaling plot of Fusarium communities in soil based on EF-1 $\alpha$ amplicon analysis. C, Stacked bar chart depicting the relative abundance of the top 21 fungal genera in soil identified by internal transcribed spacer 2 (ITS2) amplicon sequence analysis. D, Nonmetric multidimensional scaling plot of fungal communities in soil based on ITS2 amplicon sequences. 
per gram of soil (actual abundance) were significantly $(P<0.05)$ higher for susceptible strawberry than the no-plant treatment in every experiment at 52 wpi (Fig. 1B).

Soil Fusarium and fungal communities. Because we observed such poor correlations between relative and actual abundance of Fof, we adjusted the EF- $1 \alpha$ Fusarium relative abundances by the CFU of Fof per gram of soil observed at the same timepoint to obtain the estimated total abundance of each Fusarium ASV (Supplementary Fig. S5). Using this adjusted dataset, we observed that crop treatment had a very minimal effect on soil Fusarium communities (Fig. 5B); the maximum correlation for PERMANOVAs comparing any two treatments was $R^{2}=0.21$ (Supplementary Dataset S6). However, soil after the broccoli treatment had significantly lower relative abundance of the Fof ASV than the no-plant, lettuce, and susceptible strawberry treatments $\left(\log _{2} \mathrm{FC}=-2.19,-1.74\right.$, and -2.21 , respectively; $P<0.02$ ) (Supplementary Dataset S6), suggesting that it could be a good rotation crop for control of this pathogen.

More substantial treatment-level differences were apparent in soil from the analysis of ITS amplicons (Fig. 5). In addition to resulting in the lowest Fof CFU/g of soil over time (Fig. 3), the no-plant treatment appeared to be detrimental to overall Fusarium communities. This treatment had a significantly lower relative abundance of the Fusarium genus than any other treatment (adjusted $P$ value $\leq 0.001 ; \log _{2} \mathrm{FC} \geq 1.4$ ).

Culture-dependent Fusarium community profiling and comparison with amplicon-based identification of EF- $1 \alpha$ sequences. Before Fof infestation, we performed soil dilution plating of the bulk field soil used in the crop rotation experiments, isolated pure cultures of Fusarium spp. from dilution plates, and sequenced their EF- $1 \alpha$ locus. This provided a reference against which we could compare results from amplicon sequencing and confirmed that no native $F$. oxysporum isolates in the field soil shared the same EF- $1 \alpha$ haplotype as the introduced strain of Fof. From three replicate culture-dependent soil assays conducted before infestation with Fof, we recovered 93, 77, and 65 colonies of Fusarium spp. at a detection limit of $50 \mathrm{CFU} / \mathrm{g}$ soil. These 235 total colonies comprised 27 unique haplotypes. Importantly, no native $F$. oxysporum isolates in the field soil shared the same EF-1 $\alpha$ haplotype as the introduced strain of Fof. Only seven haplotypes were observed in all three samples; all others were present at low abundances (50 to $150 \mathrm{CFU} / \mathrm{g}$ ) (Fig. 6). The most abundant Fusarium sp. in this soil was F. oxysporum, which had 10 distinct haplotypes (Fig. 6).

Our analysis of EF- $1 \alpha$ amplicon sequences from roots and soil in the crop rotation experiments recovered 34 EF-1 $\alpha$ ASVs (Supplementary Fig. S6). Although amplicon sequencing was not conducted on the initial three soil samples used for culture-dependent analysis, 17 ASVs were identical to 1 of the 27 haplotypes detected by culture-dependent analysis. Of the $10 \mathrm{EF}-1 \alpha$ haplotypes that were only discovered by culture-dependent analysis, 8 were at very low abundance ( $50 \mathrm{CFU} / \mathrm{g}$; at the detection threshold) and 2 were moderately abundant (100 and $300 \mathrm{CFU} / \mathrm{g}$ ), with both in $F$. solani species complex 3. Amplicon sequence analysis recovered 17 ASVs that were not identified by culture-based haplotyping.

Conservation tillage and cover cropping significantly affected garbanzo root but not soil Fusarium community composition. We analyzed the soil Fusarium community from a long-term agricultural research experiment with four treatments: standard tillage with no off-season cover crop (STNO), standard tillage with an offseason cover crop (STCC), conservation tillage with no off-season cover crop (CTNO), and conservation tillage with an off-season cover crop (CTCC) (Fig. 1B). From this 18-year experiment, we collected roots and soil in 2017 and soil in 2018. In 2017, an earlyseason stunting and significant reduction in yield was observed in the STCC treatment; no stunting or yield differences were detected in the 2018 trial (Mitchell et al. 2021).

Despite 18 years of treatment applications, we detected few treatment-dependent differences in Fusarium community profiles. Soil Fusarium compositions were not different between years for the CTCC, CTNO, and STNO treatments. (PERMANOVA; $P \geq 0.17)$. There was a significant $\left(R^{2}=0.52 ; P=0.02\right)$ effect of year for the 2017 versus 2018 STCC soil comparison, which corresponded to the decline phenotype observed in 2017 but not 2018. With soil community data pooled across years, we observed no significant community-level differences associated with treatment $(P \geq 0.10)$ (Fig. 7A; Supplementary Dataset S7). The only four differentially abundant ASVs were associated with the STCC treatment versus all other treatments (Supplementary Dataset S7).

We observed significant differences between root Fusarium community profiles in 2017 (Supplementary Dataset S7). Interestingly, the largest effect size was observed for the treatments with differences in both tillage and cover crop practices (CTCC versus STNO; PERMANOVA $P$ value $=0.02 ; R^{2}=0.63$ ). Within treatments, the root community profiles were significantly different from soil (PERMANOVA $P$ value $\leq 0.03 ; R^{2} \geq 0.57$ ). Differential ASV abundance compared between all root and all soil samples was structured by species complex; roots were enriched for oxysporum, solani, and fujikuroi (asiaticum) ASVs, whereas soil was enriched for all incarnatum-equiseti and cyanostomum ASVs (Fig. 7C).

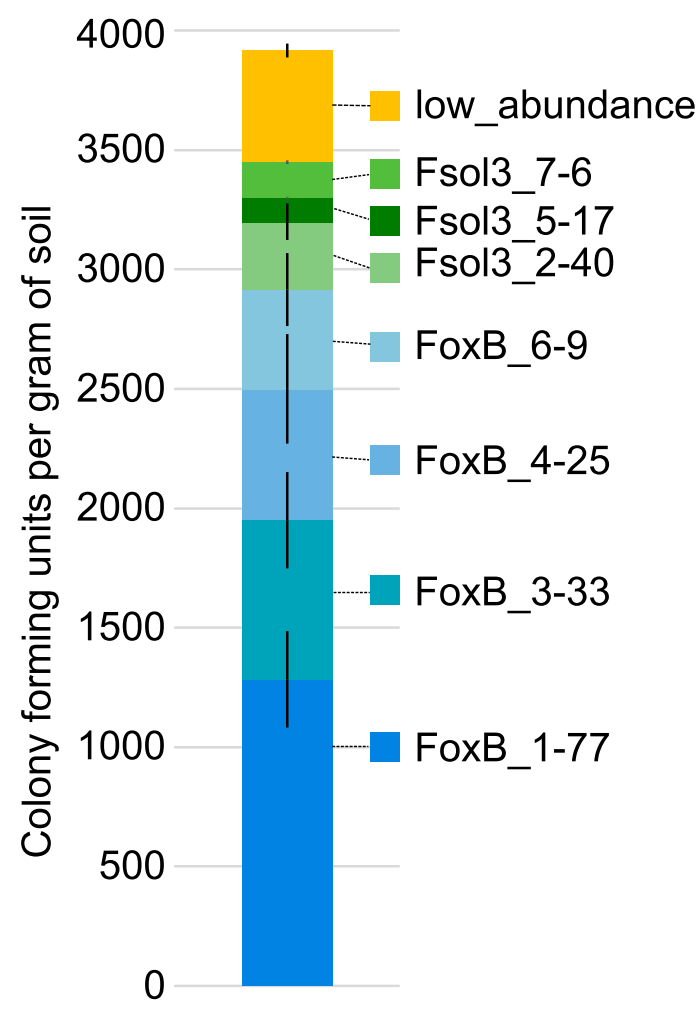

Fig. 6. Fusarium community profile of field soil before Fusarium oxysporum f. sp. fragariae (Fof) infestation at the start of the experiments. These data were determined by a culture-dependent method in which 235 Fusarium colonies were isolated in pure culture and sequenced at the translation elongation factor $1 \alpha$ locus. Haplotype IDs starting with "FoxB" are $F$. oxysporum and IDs starting with "Fsol3" are $F$. solani species complex group 3 . Haplotypes detected at a density that is $\leq 100 \mathrm{CFU} / \mathrm{g}$ of soil are considered "low abundance". All haplotype IDs are color coded and indicated to the right of their corresponding stacked bar. 


\section{DISCUSSION}

Amplicon sequencing of the EF-1 $\alpha$ locus on the Illumina MiSeq platform can achieve subspecies-level resolution among Fusarium ASVs. Incorporating the oligotyping method allowed us to decompose raw sequences into ASVs that were identical to those recovered by Sanger sequencing EF-1 $\alpha$ amplicons from pure cultures. We observed some bias with respect to over- or underreporting specific isolates. However, this bias was not as severe as the bias that has been reported for ITS primers (Bokulich and Mills 2013), and was reproducible with multiple library preparation methods and different mock communities. Some evidence of off-target amplification was observed, because not all reads could be classified to a species complex (data not shown). Despite this challenge, we obtained sufficient Fusarium sequences for our analyses. Therefore, within a study, this method should enable valid comparisons among treatments, conditions, and tissue types.

There are many potential applications for this tool, including for investigations of the biogeography of species across wide geographic regions. Studies focused on basal Fusarium clades such as the $F$. dimerum species complex would need to redesign the forward (fefF) primer; specificity toward these taxa was sacrificed for a shorter amplicon in this study. For the more terminal Fusarium clades, we demonstrate that this method can profile haplotypes with single-base-pair resolution. Because we did not detect ASVs with the same EF- $1 \alpha$ sequence as the Fof isolate that was added to the soil, we can assume that we were quantifying just the pathogenic strain when recovering its ASV. However, without this contextual knowledge, it would not be possible to assume that an ASV represents a specific Fusarium wilt pathogen; identical EF-1 $\alpha$ sequences can be shared between pathogens and nonpathogens (O'Donnell et al. 2009). This limitation is not unique to EF- $1 \alpha$; horizontal chromosome transfer in $F$. oxysporum eliminates the ability for any core genome locus to identify pathogenic strains without further contextual information (Ma et al. 2010). Nevertheless, with the appropriate experimental design, as demonstrated here, this limitation can be overcome.

Our results suggest that a causal relationship exists between plant colonization by a pathogenic strain of $F$. oxysporum (Fof, as measured by ASV relative abundance) and soil abundance after a period of tissue decomposition (measured both by ASV relative abundance and CFU per gram abundance). This inference is congruent with previous studies (Elmer and Lacy 1987; Henry et al. 2019a), and is supported by our observation that Fof had a very high relative abundance ( $>90 \%$ ) on susceptible strawberry roots (Fig. 3) and numerically highest soil inoculum densities after susceptible strawberry cultivation (Fig. 1B). This can be explained by pathogen growth on susceptible plant tissues leading to increased soil inoculum density after decomposition.

However, unexpected differences were observed between ASV relative abundance in roots and bulk soil after root decomposition. These differences indicate that treatment-dependent microbial successions can occur during plant decomposition that dilute the impact of root-inhabiting communities on subsequent soil community profiles. The outcome is that soil relative abundances after decomposition do not directly mirror those observed in root tissues. For example, the F. solani species complex 3 ASV Fsol3_5-17 had very low relative abundance in living broccoli roots but was the most abundant Fusarium ASV in soil after broccoli decomposition. This ASV also had significantly higher soil abundance after broccoli cultivation than all treatments, except for blackberry $\left(\log _{2} \mathrm{FC} \geq\right.$ 1.54; adjusted $P$ value $\leq 0.003$ ). This observation would be most

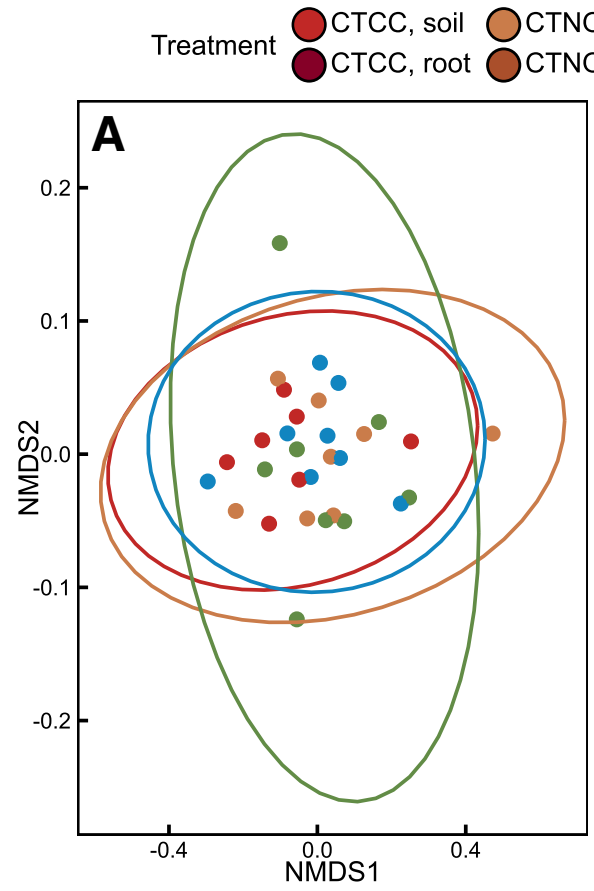

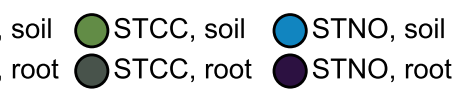

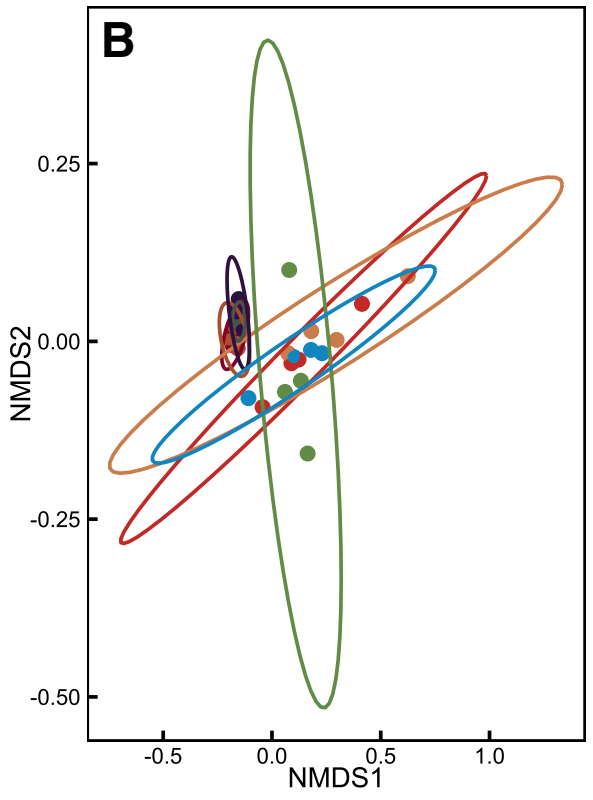

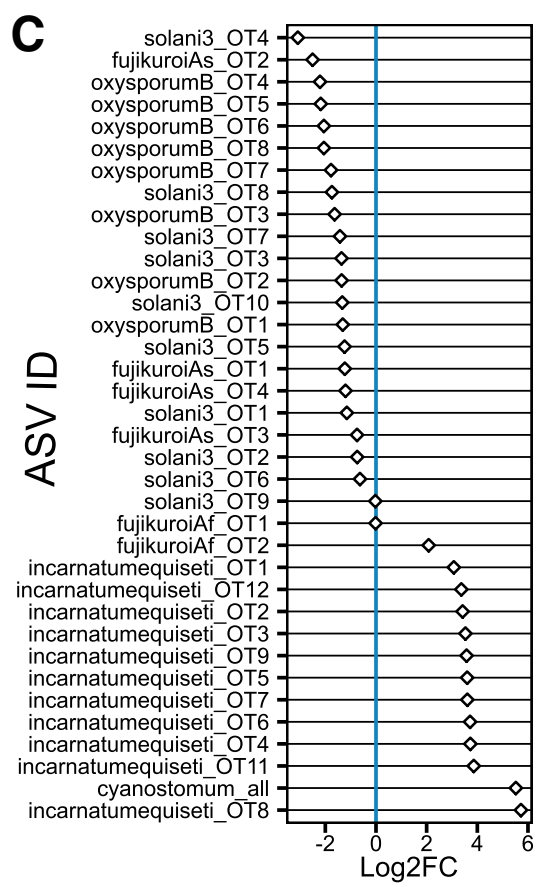

Fig. 7. Fusarium community profiles after 18-year cover-cropping or conservation-tillage field trials. A, Nonmetric multidimensional scaling plot of soil Fusarium communities in bulk soil from 2017 and 2018 organized by treatment. B, Nonmetric multi-dimensional scaling plot of root and soil Fusarium communities from 2017. C, Log $_{2}$ fold-change of Fusarium amplicon sequence variants (ASVs) between root and soil samples from 2017; positive values indicate higher proportions in root samples. Treatment codes are as follows: standard tillage with no offseason cover crop (STNO), standard tillage with an off-season cover crop (STCC), conservation tillage with no off-season cover crop (CTNO), and conservation tillage with an off-season cover crop (CTCC). 
easily explained by Fsol3_5-17 increasing in abundance during plant decomposition.

Intriguingly, despite the application of major soil disturbances in a treatment-dependent manner (such as rotation crop species, cover cropping, or conservation tillage), very little impact on bulk soil Fusarium communities was observed. For the simulated rotation crop trial, this result stood in stark contrast to the treatmentdependent effects on fungal communities measured by ITS2. The cover crop treatments at the West Side Field Station received dry biomass at an average of $0.37 \mathrm{~kg} / \mathrm{m}^{2}$ of aboveground cover crop biomass/year for the previous 18 years (Mitchell et al. 2017), and this level of input did not significantly alter the bulk soil Fusarium community.

It is possible that climatic conditions or soil physicochemical properties are greater drivers of Fusarium communities in soil than the crop-dependent effects we investigated. Edaphic characteristics such as soil $\mathrm{pH}$, texture, and mineral content can impact the severity of Fusarium wilt diseases but their impact on Fusarium community composition has not been explored (Gatch and du Toit 2017; Orr and Nelson 2018). Future research is needed to investigate additional variables that could be driving the assembly of $F$. oxysporum in agricultural environments and how these factors could impact the establishment of pathogens in new fields.

The differences we observed between relative abundances (i.e., the proportion of Fof ASV reads) and absolute abundances (i.e., CFU per gram inoculum densities) of Fof in soil highlight an important limitation of amplicon-sequencing-based methods for examining treatment effects. Any estimation of pathogen abundance derived from amplicon sequencing is expressed only as a proportion of the total microbial community, as has been noted previously (Gloor et al. 2017). Therefore, if the community varies in total abundance, true differences in pathogen absolute abundances will be obscured.

Perhaps, in part, because of successional changes in ASV abundance, our data show that, once a pathogenic strain of $F$. oxysporum establishes in soil where its susceptible host is grown, it does not necessarily become the most abundant strain of $F$. oxysporum in that field. The field soil we used for the simulated crop rotations contained 11 F. oxysporum EF-1 $\alpha$ haplotypes, as detected by a culture-dependent method. All native haplotypes were apparently nonpathogenic on the six tested crops; disease was only observed in the susceptible strawberry variety as a result of the introduced Fof strain. Although Fof was added to the soil in very high abundance at the start of the experiment (Fof at mean $=1 \times$ $10^{6} \mathrm{CFU} / \mathrm{g}$ of soil) relative to the total ASVs of $F$. oxysporum (only approximately $3 \times 10^{3} \mathrm{CFU} / \mathrm{g}$ ), Fof had a low relative abundance among $F$. oxysporum ASVs in soil by the end of 52 weeks. This result was even observed in the susceptible strawberry treatment, where it should have had the greatest competitive advantage. These data indicate that pathogenic strains, once established in soil where a susceptible host is grown, do not necessarily displace or achieve greater abundance than the native $F$. oxysporum strains.

We also observed evidence for asymptomatic host preferences between apparently nonpathogenic strains of $F$. oxysporum. Cropspecific differences in asymptomatic colonization during diseaseincompatible interactions have previously been documented for pathogenic strains of $F$. oxysporum (Dhingra and Coelho Netto 2001; Henry et al. 2019a; Leoni et al. 2013; Scott et al. 2013). This observation raises the prospect that crop rotations can be advantageous by increasing the relative abundance of possibly beneficial, nonpathogenic strains. Furthermore, beyond the binary concepts of pathogen-host and nonpathogen-host interactions, these differences point to a continuum of ability to colonize living plants. This continuum may constitute the genetic and phenotypic base upon which selection for pathogenicity is based.

In conclusion, this study shows that the community structure of Fusarium spp. in agricultural soils is remarkably static in the face of disruptions caused by crop rotation, cover crop, and tillage practices. Although a causal link may exist between reproduction on plant tissues and soil abundance, factors such as microbial succession and edaphic characteristics may play a greater role in structuring the soil Fusarium community. More work is needed to mechanistically understand how successional dynamics or soil physicochemical properties modulate the Fusarium community structure. Such knowledge could provide insights into how these processes can be leveraged to reduce pathogen abundance.

\section{ACKNOWLEDGMENTS}

We thank Lia Lopez, Michelle Haugland, Mariel Munji, and Samuel Brinker for providing technical assistance; Matthew Settles for providing technical expertise on the use of dbcAmplicons; Susan Bassein for statistical analysis of the mock communities; and the DNA Technologies Core facility at the University of California-Davis for providing unique dual-indexed primers.

\section{LITERATURE CITED}

Alabouvette, C. 1986. Fusarium wilt suppressive soils from the Chateaurenard region: Review of a 10-year study. Agronomie 6:273-284.

Bao, J., Fravel, D., Lazarovits, G., Chellemi, D. O., Berkum, P. V., and O’Neill, N. 2004. Biocontrol genotypes of Fusarium oxysporum from tomato fields in Florida. Phytoparasitica 32:9-20.

Benjamini, Y., and Hochberg, Y. 1995. Controlling the false discovery rate: A practical and powerful approach to multiple testing. J. R. Stat. Soc. Series B Stat. Methodol. 57:289-300.

Bokulich, N. A., and Mills, D. A. 2013. Improved selection of internal transcribed spacer-specific primers enables quantitative, ultra-highthroughput profiling of fungal communities. Appl. Environ. Microbiol. 79: 2519-2526

Bolwerk, A., Lagopodi, A. L., Lugtenberg, B. J. J., and Bloemberg, G. V. 2005. Visualisation of interactions between a pathogenic and beneficial Fusarium strain during biocontrol of tomato foot and root rot. Mol. Plant-Microbe Interact. 18:710-721.

Boutigny, A.-L., Gautier, A., Basler, R., Dauthieux, F., Leite, S., and Valade, R. 2019. Metabarcoding targeting the EF1 alpha region to assess Fusarium diversity on cereals. PLoS One 14:e0207988.

Brandfass, C., and Karlovsky, P. 2008. Upscaled CTAB-based DNA extraction and real-time PCR assays for Fusarium culmorum and $F$. graminearum DNA in plant material with reduced sampling error. Int. J. Mol. Sci. 9:2306-2321.

Buschmann, T., and Bystrykh, L. V. 2013. Levenshtein error-correcting barcodes for multiplexed DNA sequencing. BMC Bioinf. 14:272.

Caporaso, J. G., Bittinger, K., Bushman, F. D., DeSantis, T. Z., Andersen, G. L., and Knight, R. 2010. PyNAST: A flexible tool for aligning sequences to a template alignment. Bioinformatics 26:266-267.

Cobo-Díaz, J. F., Baroncelli, R., Le Floch, G., and Picot, A. 2019. A novel metabarcoding approach to investigate Fusarium species composition in soil and plant samples. FEMS Microbiol. Ecol. 95:1-13.

Constantin, M. E., de Lamo, F. J., Vlieger, B. V., Rep, M., and Takken, F. L. W. 2019. Endophyte-mediated resistance in tomato to Fusarium oxysporum is independent of ET, JA, and SA. Front. Plant Sci. 10:979.

Constantin, M. E., Fokkens, L., de Sain, M., Takken, F. L. W., and Rep, M. 2021. Number of candidate effector genes in accessory genomes differentiates pathogenic from endophytic Fusarium oxysporum strains. Front. Plant Sci. 12:761740.

de Lamo, F. J., Spijkers, S. B., and Takken, F. L. W. 2020. Protection to tomato wilt disease conferred by the nonpathogen Fusarium oxysporum Fo47 is more effective than that conferred by avirulent strains. Phytopathology 111: 253-257.

Demers, J. E., Gugino, B. K., and Jiménez-Gasco, M. D. M. 2015. Highly diverse endophytic and soil Fusarium oxysporum populations associated with fieldgrown tomato plants. Appl. Environ. Microbiol. 81:81-90. 
Dhingra, O. D., and Coelho Netto, R. A. 2001. Reservoir and non-reservoir hosts of bean wilt pathogen, Fusarium oxysporum f. sp. phaseoli. J. Phytopathol. 149:463-467.

Edel-Hermann, V., and Lecompte, C. 2019. Current status of Fusarium oxysporum formae speciales and races. Phytopathology 109:512-530.

Edgar, R. 2004. MUSCLE: Multiple sequence alignment with high accuracy and high throughput. Nucleic Acids Res. 32:1792-1797.

Elmer, W. H., and Lacy, M. L. 1987. Effects of crop residues and colonization of plant tissues on propagule survival and soil populations of Fusarium oxysporum f. sp. apii race 2. Phytopathology 77:381-387.

Epstein, L., Kaur, S., Chang, P. L., Carrasquilla-Garcia, N., Lyu, G., Cook, D. R., Subbarao, K., and O'Donnell, K. 2017. Races of the celery pathogen Fusarium oxysporum f. sp. apii are polyphyletic. Phytopathology 107: 463-473.

Eren, A. M., Maignien, L., Sul, W. J., Murphy, L. G., Grim, S. L., Morrison, H. G., and Sogin, M. L. 2013. Oligotyping: Differentiating between closely related microbial taxa using 16s rRNA gene data. Methods Ecol. Evol. 4: 1111-1119.

Gatch, E. W., and du Toit, L. J. 2017. Limestone-mediated suppression of Fusarium wilt in spinach seed crops. Plant Dis. 101:81-94.

Geiser, D. M., Jiménez-Gasco, M. D. M., Kang, S., Makalowska, I., Veeraraghavan, N., Ward, T. J., Zhang, N., Kuldau, G. A., and O’Donnell, K. 2004. FUSARIUM-ID v. 1.0: A DNA sequence database for identifying Fusarium. Eur. J. Plant Pathol. 110:473-479.

Gloor, G. B., Macklaim, J. M., Pawlowsky-Glahn, V., and Egozcue, J. J. 2017. Microbiome datasets are compositional: And this is not optional. Front Microbiol. 8:2224.

Gordon, T. R. 2017. Fusarium oxysporum and the Fusarium wilt syndrome. Annu. Rev. Phytopathol. 55:23-39.

Gordon, T. R., and Martyn, R. D. 1997. The evolutionary biology of Fusarium oxysporum. Annu. Rev. Phytopathol. 35:111-128.

Gordon, T. R., and Okamoto, D. 1990. Colonization of crop residue by Fusarium oxysporum f. sp. melonis and other species of Fusarium. Phytopathology 80:381-386.

Gordon, T. R., Okamoto, D., and Jacobson, D. J. 1989. Colonization of muskmelon and nonsusceptible crops by Fusarium oxysporum f. sp. melonis and other species of Fusarium. Phytopathology 79:1095-1100.

Gremme, G., Steinbiss, S., and Kurtz, S. 2013. GenomeTools: A comprehensive software library for efficient processing of structured genome annotations. IEEE/ACM Trans. Comput. Biol. Bioinf. 10:645-656.

Haware, M. P., and Nene, Y. L. 1982. Symptomless carriers of the chickpea wilt Fusarium. Plant Dis. 66:250-251.

Henry, P. M., Haugland, M., Lopez, L., Munji, M., Watson, D. C., and Gordon, T. R. 2020a. The potential for Fusarium oxysporum f. sp. fragariae, cause of Fusarium wilt of strawberry, to colonize organic matter in soil and persist through anaerobic soil disinfestation. Plant Pathol. 69:1218-1226.

Henry, P. M., Kaur, S., Pham, Q. A., Barakat, R., Brinker, S., Haensel, H., Daugovish, O., and Epstein, L. 2020b. Genomic differences between the new Fusarium oxysporum f. sp. apii (Foa) race 4 on celery, the less virulent Foa races 2 and 3, and the avirulent on celery f. sp. coriandrii. BMC Genomics 21:730.

Henry, P. M., Pastrana, A. M., Leveau, J. H. J., and Gordon, T. R. 2019a. Persistence of Fusarium oxysporum f. sp. fragariae in soil through asymptomatic colonization of rotation crops. Phytopathology 109:770-779.

Henry, P. M., Pincot, D. D. A., Jenner, B. N., Borrero, C., Avilés, M., Nam, M. H., Epstein, L., Knapp, S., and Gordon, T. R. 2021. Horizontal chromosome transfer and independent evolution drive diversification in Fusarium oxysporum f. sp. fragariae. New Phytol. 230:327-340.

Henry, P. M., Stueven, M., Miyao, E. M., Gordon, T. R., Davis, R. M., and Doan, H. K. 2019b. Genome sequence of a California isolate of Fusarium oxysporum f. sp. lycopersici race 3 , a fungus causing wilt disease on tomato. Microbiol. Resour. Announce. 8:e01713-18.

Inderbitzin, P., Ward, J., Barbella, A., Solares, N., Izyumin, D., Burman, P., Chellemi, D. O., and Subbarao, K. V. 2018. Soil microbiomes associated with Verticillium wilt-suppressive broccoli and chitin amendments are enriched with potential biocontrol agents. Phytopathology 108: 31-43.

Karlsson, I., Edel-Hermann, V., Gautheron, N., Durling, M. B., Kolseth, A., Steinberg, C., Persson, P., and Friberg, H. 2016. Genus-specific primers for study of Fusarium communities in field samples. Appl. Environ. Microbiol. 82:491-501.

Kettler, T. A., Doran, J. W., and Gilbert, T. L. 2001. Simplified method for soil particle-size determination to accompany soil-quality analyses. Soil Sci. Soc. Am. J. 65:849-852.
Komada, H. 1975. Development of a selective medium for quantitative isolation of Fusarium oxysporum from natural soils. Rev. Plant Prot. Res. 8: 114-125.

Lahti, L., and Shetty, S. 2017. Tools for microbiome analysis in R. http: $/ /$ microbiome.github.io

Leoni, C., de Vries, M., Braak, C. J. F., van Bruggen, A. H. C., and Rossing, W. A. H. 2013. Fusarium oxysporum f. sp. cepae dynamics: In-plant multiplication and crop sequence simulations. Eur. J. Plant Pathol. 137:545-561.

Love, M. I., Huber, W., and Anders, S. 2014. Moderated estimation of fold change and dispersion for RNA-seq data with DESeq2. Genome Biol. 15:550.

Ma, L., van der Does, H. C., Borkovich, K. A., Coleman, J. J., Daboussi, M., Di Pietro, A., Dufresne, M., Freitag, M., Grabherr, M., Henrissat, B., Houterman, P. M., Kang, S., Shim, W., Woloshuk, C., Xie, X., Xu, J., Antoniw, J., Baker, S. E., Bluhm, B. H., Breakspear, A., Brown, D. W., Butchko, R. A. E., Chapman, S., Coulson, R., Coutinho, P. M., Danchin, E. G. J., Diener, A., Gale, L. R., Gardiner, D. M., Goff, S., Hammond-Kosack, K. E., Hilburn, K., Hua-Van, A., Jonkers, W., Kazan, K., Kodira, C. D., Koehrsen, M., Kumar, L., Lee, Y., Li, L., Manners, J. M., Miranda-Saavedra, D., Mukherjee, M., Park, G., Park, J., Park, S., Proctor, R. H., Regev, A., Ruiz-Roldan, M. C., Sain, D., Sakthikumar, S., Sykes, S., Schwartz, D. C., Turgeon, B. G., Wapinski, I., Yoder, O., Young, S., Zeng, Q., Zhou, S., Galagan, J., Cuomo, C. A., Kistler, H. C., and Rep, M. 2010. Comparative genomics reveals mobile pathogenicity chromosomes in Fusarium. Nature 464:367-373.

Magoc, T., and Salzberg, S. L. 2011. FLASH: Fast length adjustment of short reads to improve genome assemblies. Bioinformatics 27:2957-2963.

McMurdie, P. J., and Holmes, S. 2013. phyloseq: An R package for reproducible interactive analysis and graphics of microbiome census data. PLoS One 8:e61217.

Mitchell, J. P., Shreshta, A., Epstein, L., Dahlberg, J. A., Ghezzehei, T., Araya, S., Richter, B., Kaur, S., Henry, P. M., Munk, D. S., Light, S., Bottens, M., and Zaccaria, D. 2021. No-tillage sorghum and garbanzo yields match or exceed standard tillage yields. Calif. Agric. 75:112-120.

Mitchell, J. P., Shreshta, A., Mathesius, K., Scow, K. M., Southard, R. J., Haney, R. L., Schmidt, S. M., Munk, D. S., and Horwath, W. R. 2017. Cover cropping and no-tillage improve soil health in an arid irrigated cropping system in California's San Joaquin Valley, USA. Soil Tillage Res. 165:325-335.

Mol, L., Scholte, K., and Vos, J. 1995. Effects of crop rotation and removal of crop debris on the soil population of two isolates of Verticillium dahliae. Plant Pathol. 44:1070-1074.

O’Donnell, K., Gueidan, C., Sink, S., Johnston, P. R., Crous, P. W., Glenn, A., Riley, R., Zitomer, N. C., Colyer, P., Waalwijk, C., van der Lee, T., Moretti, A., Kang, S., Kim, H., Geiser, D. M., Juba, J. H., Baayen, R. P., Cromey, M. G., Bithell, S., Sutton, D. A., Skovgaard, K., Ploetz, R., Kistler, H. C., Elliott, M., Davis, M., and Sarver, B. A. J. 2009. A two-locus DNA sequence database for typing plant and human pathogens within the Fusarium oxysporum species complex. Fungal Genet. Biol. 46:936-948.

O’Donnell, K., Kistler, H. C., Cigelnik, E., and Ploetz, R. 1998. Multiple evolutionary origins of the fungus causing Panama disease of banana: Concordant evidence from nuclear and mitochondrial gene genealogies. Proc. Nat. Acad. Sci. U.S.A. 95:2044-2049.

O’Donnell, K., Rooney, A. P., Proctor, R. H., Brown, D. W., McCormick, S. P., Ward, T. J., Frandsen, R. J. N., Lysøe, E., Rehner, S. A., Aoki, T., Robert, V. A. R. G., Crous, P. W., Groenewald, J. Z., Kang, S., and Geiser, D. M. 2012. Phylogenetic analyses of $R P B 1$ and $R P B 2$ support a middle Cretaceous origin for a clade comprising all agriculturally and medically important fusaria. Fungal Genet. Biol. 52:20-31.

Orr, R., and Nelson, P. N. 2018. Impacts of soil abiotic attributes on Fusarium wilt, focusing on bananas. Appl. Soil Ecol. 132:20-33.

Peralta, A. L., Sun, Y., McDaniel, M. D., and Lennon, J. T. 2018. Crop rotational diversity increases disease suppressive capacity of soil microbiomes. Ecosphere 9:e02235.

R Core Team. 2013. R: A Language and Environment for Statistical Computing. R Foundation for Statistical Computing, Vienna, Austria.

Schneider, R. W. 1984. Effects of nonpathogenic strains of Fusarium oxysporum on celery root infection by $F$. oxysporum f. sp. lini and novel use of the Lineweaver-Burk double reciprocal plot technique. Phytopathology 74 : 646-653.

Scott, J. C., Gordon, T. R., Kirkpatrick, S. C., Koike, S. T., Matheron, M. E., Ochoa, O. E., Truco, M. J., and Michelmore, R. W. 2012. Crop rotation and genetic resistance reduce risk of damage from Fusarium wilt in lettuce. Calif. Agric. 66:20-24.

Scott, J. C., McRoberts, N., and Gordon, T. R. 2013. Colonization of lettuce cultivars and rotation crops by Fusarium oxysporum f. sp. lactucae, the cause of Fusarium wilt of lettuce. Plant Pathol. 63:548-553. 
Siegel-Hertz, K., Edel-Hermann, V., Chapelle, E., Terrat, S., Raaijmakers, J. M., and Steinberg, C. 2018. Comparative microbiome analysis of a Fusarium wilt suppressive soil and a Fusarium wilt conducive soil from the Châteaurenard Region. Front. Microbiol. 9:568.

Singh, S. K., Nene, Y. L., and Reddy, M. V. 1990. Influence of cropping systems on Macrophomina phaseolina populations in soil. Plant Dis. 74: 812-814.

Taylor, D. L., Walters, W. A., Lennon, N. J., Bochicchio, J., Krohn, A., Caporaso, J. G., and Pennanen, T. 2016. Accurate estimation of fungal diversity and abundance through improved lineage-specific primers optimized for Illumina amplicon sequencing. Appl. Environ. Microbiol. 82: 7217-7226.

Turenne, C. Y., Sanche, S. E., Hoban, D. J., Karlowsky, J. A., and Kabani, A. M. 1999. Rapid identification of fungi by using the ITS2 genetic region and an automated fluorescent capillary electrophoresis system. J. Clin. Microbiol. 37:1846-1851.
Weller, D. M., Raaijmakers, J. M., McSpadden Gardener, B. B., and Thomashow, L. S. 2002. Microbial populations responsible for specific soil suppressiveness to plant pathogens. Annu. Rev. Phytopathol. 40:309-348.

White, T. J., Bruns, T. D., Lee, S., and Taylor, J. 1990. Amplification and direct sequencing of fungal ribosomal RNA genes for phylogenetics. Pages 315322 in: PCR Protocols: A Guide to Methods and Applications. M. A. Innis, D. H. Gelfand, J. J. Sninsky, and T. H. White, eds. Academic Press, San Diego, CA.

Williams, J. E., Carrothers, J. M., Lackey, K. A., Beatty, N. F., York, M. A., Brooker, S. L., Shafii, B., Price, W. J., Settles, M. L., McGuire, M. A., and McGuire, M. K. 2017. Human milk microbial community structure is relatively stable and related to variations in macronutrient and micronutrient intakes in healthy lactating women. J. Nutr. 147:1739-1748.

Xiao, C. L., Subbarao, K. V., Schulbach, K. F., and Koike, S. T. 1998. Effects of crop rotation and irrigation on Verticillium dahliae microsclerotia in soil and wilt in cauliflower. Phytopathology 88:1046-1055. 